\title{
On the Scalability of Dynamic Flex-Grid/SDM Optical Core Networks
}

\author{
Rubén Rumipamba-Zambrano, Jordi Perelló, Joan M. Gené, and Salvatore Spadaro
}

Advanced Broadband Communications Center (CCABA), Universitat Politècnica de Catalunya (UPC), Jordi Girona 1-3, 08034 Barcelona, Spain, e-mail: rrumipam@ac.upc.edu

\begin{abstract}
Future optical core networks realizing Flex-Grid and Space Division Multiplexing (Flex-Grid/SDM) will be key to effectively cope with the rapid growth of the Internet traffic, thanks to their superior capacity and spectrum utilization flexibility. Specifically about SDM, the spatial dimension can be considered either fixed or flexible. The goal of this work is two-fold. Firstly, it aims at quantifying the capacity (in terms of throughput) of Flex-Grid/SDM networks assuming either a fixed or a flexible spatial dimension. To this end, we scale up the spatial multiplicity from 7 to 30 spatial channels, considering MultiFiber (MF) and Multi-Core Fiber (MCF) solutions. Secondly, it also aims at analyzing the throughput scalability in these networks versus their hardware requirements, especially from the transponder requirements point of view. The obtained results show that, for a $4.3 \mathrm{x}$ spatial multiplicity scaling factor, the throughput of spatially fixed Flex-Grid/SDM networks scales up to $3.5 x$. Conversely, when the spatial dimension is considered flexible, throughput can be pushed up to a $5 x$ factor for both MF and MCF solutions. However, a longterm realization of spectrally-spatially flexible optical networks (SS-FONs) requires up to $9.6 x$ more laser sources and $2.7 x$ electronic complexity (per required connection) in the transponders compared to spatially fixed FlexGrid/SDM networks. Furthermore, SS-FONs enable different options for assigning the spectral and spatial resources to traffic demands. In this sense, another contribution of this work is the introduction of a novel methodology that trades network throughput for transponder (the major network equipment cost in SDM networks) complexity reduction.
\end{abstract}

\section{List of acronyms and nomenclature}

$\begin{array}{ll}\alpha & \text { Weighting parameter } \\ \alpha_{i} & \text { Highest } \alpha \text { which yields the same throughput as } \alpha=0 \\ \alpha_{o p t} & \text { Weighting parameter which yields the highest throughput } \\ b & \text { Function that weights spectral and spatial resources } \\ \beta & \text { Set of } b \text { values } \\ \text { BBP } & \text { Bandwidth Blocking Probability } \\ \text { BV-OXC } & \text { Bandwidth Variable Optical Cross Connect } \\ \text { BVT } & \text { Bandwidth Variable Transponder } \\ \text { DT12 } & \text { 12-node Deutsche Telekom Optical Network } \\ d & \text { Demand } \\ \text { e2e-grooming } & \text { end-to-end spatial traffic grooming } \\ \Delta_{f s} & \text { Frequency Slot width }\end{array}$

(C) 2018 Elsevier. This manuscript version is made available under the CC-BY-NC-ND 4.0 license http://creativecommons.org/licenses/by-nc-nd/4.0/ 


\begin{tabular}{|c|c|}
\hline $\mathrm{F}$ & Set of frequency slots per spatial channel \\
\hline FJoS & Fractional Joint Switching \\
\hline FMF & Few-Mode Fiber \\
\hline FM-MCF & Few-Mode Multi-Core Fiber \\
\hline FNB & Fully Non-Blocking \\
\hline FS & Frequency Slot \\
\hline GB & Guard band \\
\hline GoS & Grade-of-Service \\
\hline HT & Holding Time \\
\hline IAT & Inter-Arrival Time \\
\hline ICXT & Inter-Core crosstalk \\
\hline InS with LC & Independent Switching with Lane Change support \\
\hline InS w/o LC & Independent switching without Lane Change support \\
\hline JoS & Joint Switching \\
\hline K & $\begin{array}{l}\text { Number of candidate physical paths over which a lightpath } \\
\text { can be established }\end{array}$ \\
\hline Ip & lightpath \\
\hline Lp & Set of active lightpaths \\
\hline $\operatorname{Lp}_{s, t}$ & $\begin{array}{l}\text { Set of active lightpaths with source node } s \text { and destination } \\
\text { node } t\end{array}$ \\
\hline M & Set of modulation formats \\
\hline MC & Multi-carrier \\
\hline MCF & Multi-Core Fiber \\
\hline MIMO & Multiple-Input Multiple-Output \\
\hline MF & Multi-Fiber \\
\hline MMF & Multi-Mode Fiber \\
\hline$n$ & Number of bits per symbol \\
\hline$n_{O C}$ & Number of optical carriers needed to serve $d$ \\
\hline$\overline{n_{O C}}$ & Mean number of optical carriers needed to serve $d$ \\
\hline$n_{s}$ & Number of spatial channels needed to serve $d$ \\
\hline$n_{f s}$ & Number of frequency slots needed to serve $d$ \\
\hline$\overline{n_{T R x}}$ & Mean number of transceivers per super-channel \\
\hline NWDM & Nyquist-Wavelength Division Multiplexing \\
\hline$\Omega$ & Set of candidate super-channel configurations \\
\hline OC & Optical carrier \\
\hline PM & Polarization Multiplexing \\
\hline$r$ & Mean bit-rate per connection \\
\hline & Requested bit-rate of demand $d$ \\
\hline ROADM & Reconfigurable Optical Add Drop Multiplexer \\
\hline RMSSA & $\begin{array}{l}\text { Route, Modulation Format, Space and Spectrum } \\
\text { Assignment }\end{array}$ \\
\hline$R_{S}$ & Mean symbol rate per transmitter \\
\hline$S$ & Set of spatial channels per link \\
\hline $\mathrm{Sb}-\mathrm{Ch}$ & Sub-Channel \\
\hline SC & Single-carrier \\
\hline $\mathrm{SCh}$ & Super-Channel \\
\hline SDM & Space Division Multiplexing \\
\hline$s_{d}$ & Source node of demand $d$ \\
\hline$S E$ & Spectral Efficiency \\
\hline SMF & Single Mode Fiber \\
\hline SNR & Signal-to-Noise-Ratio \\
\hline Spa-SCh & Spatial Super-Channel \\
\hline
\end{tabular}




$\begin{array}{ll}\text { Spe-SCh } & \text { Spectral Super-Channel } \\ \text { S2-SCh } & \text { Spectral-Spatial Super-Channel } \\ \text { SP } & \text { Shortest Path } \\ \text { SS-FON } & \text { Spectrally-Spatially Flexible Optical Network } \\ \text { SSS } & \text { Spectrum Selective Switch } \\ t_{d} & \text { Destination node of demand } d \\ \text { TP } & \text { Traffic Profile } \\ \text { TR } & \text { Transmission Reach } \\ \text { US26 } & \text { 26-node US Optical Network } \\ \text { WDM } & \text { Wavelength Division Multiplexing }\end{array}$

\section{Introduction}

Flex-Grid [1] and Space Division Multiplexing (SDM) [2] technologies have arisen large interest of the research community in the recent years, on the way to find out novel solutions that optimize the spectrum usage and overcome the capacity limit of the single mode fibers (SMFs), bounded by the so-called non-linear Shannon's limit.

Flex-Grid discretizes the optical spectrum in frequency slots (FSs) of $12.5 \mathrm{GHz}$ width, as recommended by the ITU-T in [3]. Unlike Wavelength Division Multiplexing (WDM) networks, Flex-Grid allows transmissions at flexible bit-rates thanks to the programmability of several key parameters, such as the modulation format, symbol rate, and sub-channel multiplicity, among others. Ultra-high bit-rate transmissions are possible by the concatenation of multiple adjacent sub-channels (Sb-Chs), thus forming a super-channel ( $\mathrm{SCh}$ ). These programmable functions are supported by several network elements, like the Bandwidth Variable Transponders (BVTs) that transmit/receive the optical signals based on single-carrier (SC) or multi-carrier (MC) systems. The transmitted optical signals are subsequently switched and filtered at intermediate Bandwidth Variable Optical Cross Connect (BV-OXC) network nodes. In particular, BV-OXCs with additional add/drop capabilities for connections that originate/terminate at the network node are implemented as Reconfigurable Optical Add/Drop Multiplexers (ROADMs), based on Spectrum Selective Switches (SSSs).

In turn, SDM is considered as the "next-frontier" of fiber optics, able to scale up the capacity of current WDM optical fiber systems [4]. Different SDM flavors able to multiply the number of available spatial channels are currently under research. As a starting point, SMFs of current telecom operators can be upgraded to bundles of parallel SMFs, acting as a Multi-Fiber (MF) solution. However, novel fiber designs [5] are also attractive to facilitate system integration with SDM. For example, the spatial multiplicity can rely on the transmission of different fiber modes; such is the case of Multi-Mode Fibers (MMFs) or Few-Mode Fibers (FMFs). Another alternative solution is to increase the number of cores within each fiber cladding, typically referred to as Multi-Core Fibers (MCFs). Moreover, if these cores carry few modes each, this kind of fibers is called Few-Mode Multi-Core Fibers (FM-MCFs). The coupling between modes or cores is a new impairment to be considered, in order to determine if equalization, typically based on Multiple-Input and Multiple-Output (MIMO) processing is necessary. MCFs have become particularly attractive given their extremely low inter-core crosstalk (ICXT) values [6][10], avoiding the need of MIMO equalization, even over long-haul transmissions. This type of fibers is referred to as weakly-coupled MCFs, for which we estimated in our previous work [11] 
the transmission reach (TR) as the most restrictive limitation, either imposed by Signal-toNoise-Ratio (SNR) or ICXT.

Channel allocation is an important aspect in offline and online scenarios, so as to maximize the network Grade-of-Service (GoS). To perform this task in SDM networks, apart from the transmission media, another key aspect to be considered is the switching strategy of the multiplexed channels at the ROADM devices. Several SDM-ROADM designs have been proposed in the literature, aimed at relaxing the hardware requirements and dealing with ICXT [12]-[14]. The most flexible, but also the most expensive one is the so-called Independent Switching (InS) with Lane Change (LC) support [12] or Fully Non-Blocking (FNB) in [14]. This SDM-ROADM architecture provides full interconnection flexibility between ports and spatial channels (fibers/cores/modes). Thus, InS with LC support SDM-ROADMs allow any spectral portion from any spatial channel and input port to be independently directed to any spatial channel at any output port. The spatial switching capability can be limited (i.e., the so-called InS without LC change support [12]) aimed at reducing the node complexity with minimal throughput penalties, as demonstrated in [14]. Since InS (w/ or w/o LC change support) switches optical signals with spectral and spatial granularity, it is compatible with uncoupled or weakly-coupled SDM fibers. A more restrictive (but less expensive) alternative switching strategy is the so-called Joint Switching (JoS) [12], [15], which jointly switches a certain spectrum portion in all spatial channels at once. All spatial channels are treated as a single entity and this technique is mandatory to be applied with strongly-coupled SDM fibers. An intermediate solution between InS and JoS, called Fractional Joint Switching (FJoS), is also possible, where the spatial channels are grouped into subgroups and JoS is independently applied to any of them. In FJoS, the smaller the subgroup size, the higher the flexibility in the spatial domain. This switching strategy is also suitable for uncoupled SDM fibers. Recent work [16] includes an analysis of the node complexity and cost of all aforementioned switching architectures, while [17] surveys different schemes for resource allocation in Spectrally and Spatially Flexible Optical Networks (SS-FONs [18]).

Considering the aforementioned aspects, different SCh allocation policies are possible with the introduction of the space dimension [19]. Indeed, SCh configuration depends on how its SbChs are arranged across both spectral and spatial domains. Sb-Chs of a spectral super-channel (Spe-SCh) shall be contiguously allocated across the spectral domain over single spatial channel using MC systems, whereas those of a spatial super-channel (Spa-SCh) shall be allocated across the spatial domain over a SC system. The accommodation of multiple Sb-Chs across both spatial and spectral domains (S2-SCh) is also feasible in SDM networks. Results reported in [20] show that Spe-SChs may yield better performance (in terms of Bandwidth Blocking Probability, BBP), as they require much less spectral overhead due to Guard Bands (GBs). GBs between Sb-Chs can be eliminated by employing Nyquist-Wavelength Division Multiplexing (NWDM) [21], whilst GBs between SChs are always necessary. Nevertheless, the allocation of Spa-SChs together with the application of the JoS technique can potentially reduce the cost in terms of hardware components. Regarding S2-SCh, reference [22] proposes algorithms for their configuration in static scenarios considering transceivers with fixed symbol rate, so may cause spectral wastage by rounding the bit-rate of demands. To the best of our knowledge, no study is available in the literature evaluating the performance and appropriateness of these intermediate configurations between Spe-SChs and Spa-SChs in 
dynamic scenarios, which should yield an interesting trade-off between performance and cost in SS-FONs.

In this paper, we evaluate the scalability of Flex-Grid/SDM networks by increasing the spatial multiplicity $|S|$ from 7 to 30 spatial channels. To this end, we consider both options for the space dimension, namely, spatially fixed and spatially flexible. We also contemplate several MCF prototypes available in the literature, as well as equivalent MF solutions. For SS-FONs, we propose a novel methodology to select the proper SCh configuration. Moreover, we analyze the hardware requirements of spatially fixed Flex-Grid/SDM networks and SS-FONs (in terms of BVTs especially). The rest of the paper is organized as follows. Section 2 presents the different $\mathrm{SCh}$ allocation policies in two separated subsections. Subsection 2.1 describes the SCh allocation in spatially fixed Flex-Grid/SDM networks, whilst subsection 2.2 describes it assuming flexibility in both spectral and spatial domains, i.e., in SS-FONs. Numerical results are shown in section 3, which is also divided in three subsections. Subsection 3.1 presents the network scenario that we use to evaluate the scalability of Flex-Grid/SDM networks. Next, subsection 3.2 presents the throughput scalability results of spatially fixed Flex-Grid/SDM networks, whereas subsection 3.3 shows the results for SS-FONs. Finally, the main conclusions of this study and future research lines are reported in section 4.

\section{Super-channel Allocation}

As mentioned in the previous section, different SChs allocation policies are possible, depending on how Sb-Chs are distributed over the spectral and spatial domains. Moreover, the spectral and spatial resources assigned to these SChs depend on whether both dimensions are fixed or flexible. In our study, we always consider flexibility in the spectral domain, while for the spatial dimension, we contemplate both options, namely, fixed (mid-term SDM horizon) and flexible (long-term SDM horizon), as explained in the following subsections 2.1 and 2.2, respectively.

\subsection{SCh allocation in spatially fixed Flex-Grid/SDM networks}

Similarly to the rigid spectral grid assigned to channels in traditional WDM networks, the spatially fixed term refers to allocation of a rigid number of spatial channels (e.g., all spatial channels, $|S|$ ) to every connection. This means that, the direct allocation policy to be applied is Spa-SChs, where each spatial channel allocates one fraction of the required bandwidth. In this context, let us explain some concepts behind Spa-SChs. A Spa-SCh can be formed by a sharedlaser source (this implies one unique central frequency for all Sb-Chs) and a certain number of transceivers (e.g., Dual Polarization in-phase/quadrature Modulators -PM I/Q Mod- and coherent detectors) per $\mathrm{Sb}$-Ch. The maximum symbol rate of the transceivers will determine whether the Spa-SCh is based on SC or MC system (which can also be seen as S2-SCh). Indeed, if the bit-rate per spatial channel exceeds the symbol_rate $\times n$ product, where $n$ is the number of bits per symbol of the modulation format, more than one $\mathrm{OC}$ will be required. Fig. 1 shows two examples of Spa-SChs with SC [Fig. 1(a)] and MC [(Fig. 1(b)] system with the transmitter part (i.e., PM I/Q Mod) of one BVT. Each transmitter modulates a fraction of the original demand that comes from a Digital-to-Analog Converter (DAC). Besides, Guard-Bands (GBs) must be added to each Sb-Ch (represented only as "Sb" in Fig. 1 for simplicity) or group of Sb-Chs per spatial channel to allow SSS filters to properly capture the incoming optical 
signals. Each $O C$ at frequency $f_{i}$ is generated by one laser source, shared by several transmitters. GBs between Sb-Chs of the same SCh can be avoided if ideal NWDM is assumed.

Furthermore, the allocation of the Sb-Chs (of one Spa-SCh) in the same spectrum portion across all spatial channels allows they can be switched together at once. This switch technique is called JoS, as introduced before, and has been demonstrated in [15]. Even though the switching is realized across all spatial channels $(|S|)$, the optimal allocation of one traffic demand may only require some of them in reality $\left(n_{s}\right)$, depending on the demand bit-rate $r_{d}$. Consequently, the unallocated spatial channels cause spectrum wastage. Another particularity of spatially fixed Flex-Grid/SDM networks (with JoS) is that, since the allocated spectral resources (i.e., the number of FSs, $n_{f s}$ ) are the same across all spatial channels, the routing problem is equivalent to Flex-Grid over a single spatial channel. The goal is to assign enough $n_{S}$ in order to minimize the $n_{f s}$ allocated to each spatial channel, thus maximizing, therefore, the GoS of the network. $n_{s}$ can take any value in the interval $1 \leq n_{s} \leq|S|$. The largest demands (in terms of bit-rate) could require allocating even $|S|$, while the smaller ones could require just one spatial channel in order to obtain the lowest $n_{f s}$ value. The minimum $n_{f s}$ is ensured when $r_{d}$ is split over the entire $|S|$ (i.e., initially $n_{s}=|S|$ ) according to Eq. (1):

$$
n_{f s}=\left\lceil\frac{r_{d} /\left(n_{s} \cdot S E\right)+G B}{\Delta_{f s}}\right\rceil
$$

where $S E$ accounts for the Spectral Efficiency of the selected modulation format in $\mathrm{b} /(\mathrm{s} \cdot \mathrm{Hz})$; and $\Delta_{f s}$ the FS width (i.e., $12.5 \mathrm{GHz}$ according to ITU-T recommendation). Due to the grid restrictions, i.e., the integrality constraint imposed to $n_{f s}$, the initial $n_{s}$ value may be reduced while remaining $n_{f s}$ unaffected, as in the Partial Core Assignment (PCA) strategy detailed in [11]. As an example, let us consider $|S|=7, \mathrm{~GB}=7.5 \mathrm{GHz}, \Delta_{f s}=12.5 \mathrm{GHz}$ and flexible symbol rate with maximum value of $32 \mathrm{GBaud}$. Taking into account these parameters in Fig. 1(a) we have a $400 \mathrm{~Gb} / \mathrm{s}$ demand with PM-16QAM, then $n_{f s}=2$ with the initial $n_{s}=7$. However, we could reduce $n_{s}$ down to 3 (spatial channels from $s_{1}$ to $s_{3}$ ), while $n_{f s}$ remains equal to 2 . In this example, we only need one OC shared by 3 transmitters operating at 16.67 GBaud $\left[400 \mathrm{~Gb} / \mathrm{s} \div\left(3 \cdot 8 \frac{\text { bits }}{\text { symbol }} \cdot 1 \mathrm{OC}\right)\right]$. In Fig. $1(\mathrm{~b})$, we have a $2 \mathrm{~Tb} / \mathrm{s}$ demand employing PM16QAM. So, $n_{f s}=4$ with the initial $n_{s}=7$ value, which can be reduced to 6 (spatial channels from $s_{1}$ to $s_{6}$ ), remaining $n_{f s}$ equal to 4 . As the maximum symbol rate is $32 \mathrm{GBaud}$, this time we need two OCs, each one shared by 6 transmitters operating at 20.83 GBaud [ $2 \mathrm{~Tb} / \mathrm{s} \div(6$. $8 \frac{\text { bits }}{\text { symbol }} \cdot 2$ OCs)]. 


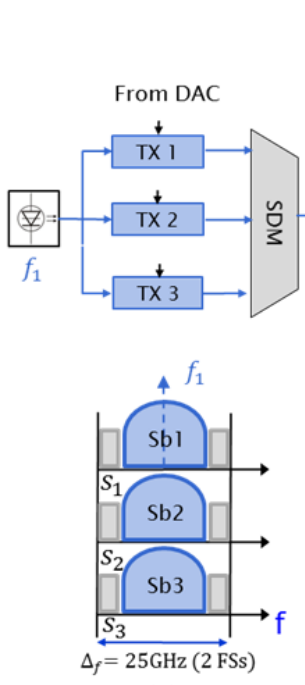

(a)
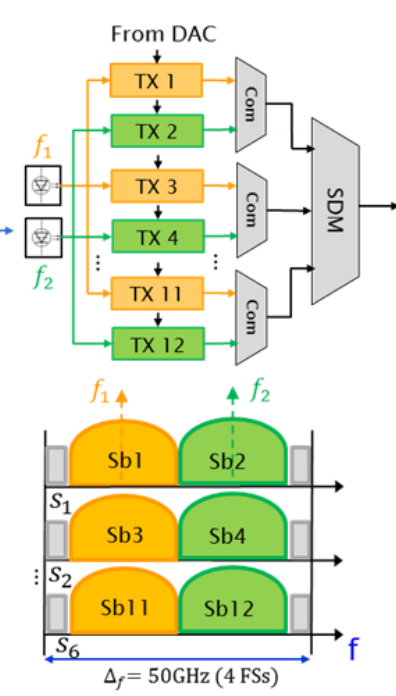

(b)
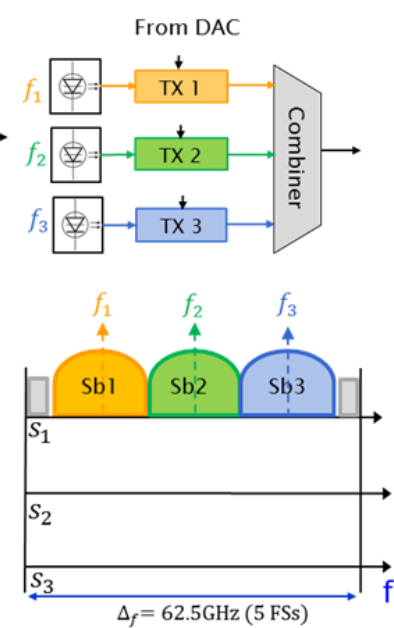

(c)

Fig. 1. Simplified version of BVT for spatial super-channel [with (a) SC, (b) MC] and spectral superchannel (c).

In the previous example in Fig. 1(a) 2 FSs were assigned in 3 (out of 7) spatial channels for a $400 \mathrm{~Gb} / \mathrm{s}$ demand. Therefore, the unallocated spatial channels $\left(n_{s}^{\prime}=|S|-n_{s}\right)$ were 4 . If we employ directly the JoS technique, we are wasting $2 \mathrm{FSs} \times 4=8 \mathrm{FSs}$. An alternative to increase the spectrum usage would be to reuse the active lightpaths in such a way that the free spectrum portions of the unused spatial channels can be assigned to other demands that share the same source $\left(s_{d}\right)$ and destination $\left(t_{d}\right)$ nodes. This can be called end-to-end spatial traffic grooming (e2e-grooming), similarly as the technique called full-match resource reuse in [23], without modifying the ROADM architecture for JoS and only requiring flexibility in the $s_{d}$ and $t_{d}$ nodes to aggregate/disaggregate traffic to the active lightpaths. One lightpath $(I p)$ can be reused as many times as needed until filling all spatial channels, all of them switched together using JoS.

To illustrate e2e-grooming, let us consider the example in Fig. 2. In this case, demands $d_{1}, d_{3}$ and $d_{4}$, that is, (400 Gb/s, PM-16AM), (100 Gb/s, PM-64QAM) and (100 Gb/s, PM-64QAM), respectively, are co-routed because they share $s_{d}$ and $t_{d}$ nodes. Therefore, if the available and required spatial resources allow it, they can share the same lightpath established for demand $d_{1}(I p 1)$. Note that, all signals that share Ip1 are modulated over the same OC at central frequency $f_{i}$. Meanwhile, demand $d_{2}$ has to establish a new lightpath $1 p 2$ because it has different $s_{d}$ and $t_{d}$ nodes. In this example, the $2 \times 100 \mathrm{~Gb} / \mathrm{s}$ demands reuse $I p 1$, hence reducing the spatial wastage from 4 to 0 (7/7 spatial channels are occupied). Meanwhile, the spectrum wastage is reduced from $8 \mathrm{FSs}$ to $4 \mathrm{FSs}$ (because $I p 1$ is shared by different demands in terms of bit-rate and, therefore, the number of required FSs also differs).

In general, when a new demand $d_{i}$ arrives at the network, first we should find out in the set of active lighpaths $(L p)$ the ones that share the same source and destination nodes as $d_{i}$ $\left(L p_{s, t} \subseteq L p\right)$ and check if any of them has enough spectral and spatial resources available to be assigned to $d_{i}$; otherwise, a new lightpath $I p$ has to be established. To this end, $\mathrm{K}=3$ Shortest Paths (SPs) are used as feasible candidate paths. For each candidate path $(p)$, the most efficient modulation format, with a TR equal or higher than the physical path length, is selected. Then, the optimal amount of required spatial and spectral resources $\left(n_{s}, n_{f s}\right)$ are 
determined by using PCA strategy. Finally, if there exist $n_{f s}$ free FSs in the available spectrum ( $F$ : set of FSs) of the first spatial channel along $p$, demand $d_{i}$ is allocated. Otherwise, the subsequent candidate paths are analyzed. If no candidate path can allocate $d_{i}$, it is finally blocked. Pseudo-code 1 shows a greedy heuristic to solve the Route, Modulation Level, Space, and Spectrum Assignment (RMSSA) problem for spatially fixed Flex-Grid/SDM optical networks. This heuristic is an updated version of the one presented in our previous work [24] with the TR estimations presented in [11]. Pseudo-code 2 shows the e2e-grooming operation, which can be incorporated to the RMSSA heuristic.

The time complexity of the RMSSA heuristic described above can be analyzed as follows. In the worst-case, the allocation of a lightpath includes searching free spatial-spectral resources over all established lightpaths (for e2e-grooming operation) and overall K-SPs. Specifically, for the e2e-grooming operation (Pseudo-code 2), its time complexity is the sum of finding, over $|L p|$ established lightpaths, those ones whose source $(s)$ and destination $(t)$ nodes are equal to the ones of $d_{i}$ plus the time complexity of sorting $L p_{s, t}$ in ascending order (to do this, the wellknown Timsort procedure is used, therefore, the time complexity of this procedure is $\left.\left|L p_{s, t}\right| \cdot \log \left|L p_{s, t}\right|\right)$ and plus the time complexity of selecting the most efficient modulation format (from $|M|$ possible ones) together with the computation of the optimal spatial-spectral resources per SCh (by using PCA strategy, $\mathcal{O}(|S|))$ for each $l p \in L p_{s, t}$. Then, the overall time complexity of the e2e-grooming operation is equal to $\mathcal{O}\left(|L p|+\left|L p_{s, t}\right| \cdot \log \left|L p_{s, t}\right|+\right.$ $\left.\left|L p_{s, t}\right|(|M|+|S|)\right)$. As for the time complexity of searching for free spatial-spectral resources over K-SPs (Pseudo-code 1), firstly, the K-SPs are computed by using the Yen's algorithm [25], i.e., the time complexity of this step is equal to $\mathcal{O}(K|V|(|E|+|V| \cdot \log |V|))$, where $|V|$ and $|E|$ account for the number of nodes and links in the network, respectively. To this time complexity has to be added the one of selecting the modulation format together with the one of the PCA strategy, plus the time complexity of searching for free spectral resources in $F$ over up to $|E|$ links for each $p \in P$, i.e., $\mathcal{O}(|P|(|M|+|S|+|F||E|))$. Note that, for a new lightpath, the spectral assignment needs searching only in the $F$ of the first spatial channel per link. Finally, the overall time complexity per arrival connection of this RMSSA heuristic is $\mathcal{O}\left(|L p|+\left|L p_{s, t}\right|\left(\log \left|L p_{s, t}\right|+|M|+|S|\right)+K|V|(|E|+|V| \cdot \log |V|)+|P|(|M|+|S|+\right.$ $|F||E|))$. In the best case, the computational complexity would only include the searching of free spatial-spectral resources over the already established lightpaths (i.e., successful e2egrooming operation). 


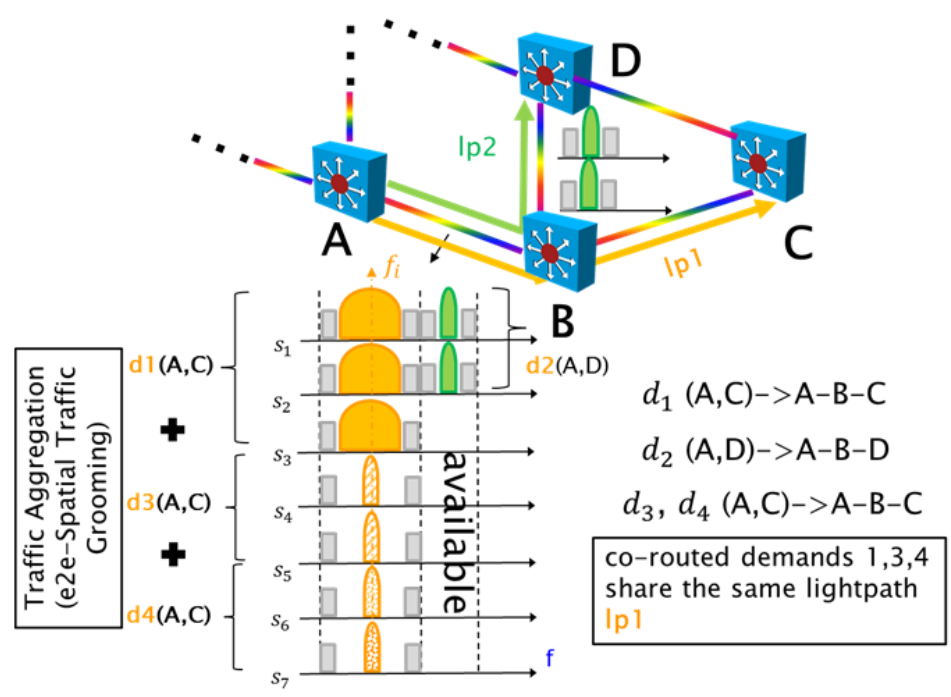

Fig. 2. Spatial super-channel allocation with e2e-grooming.

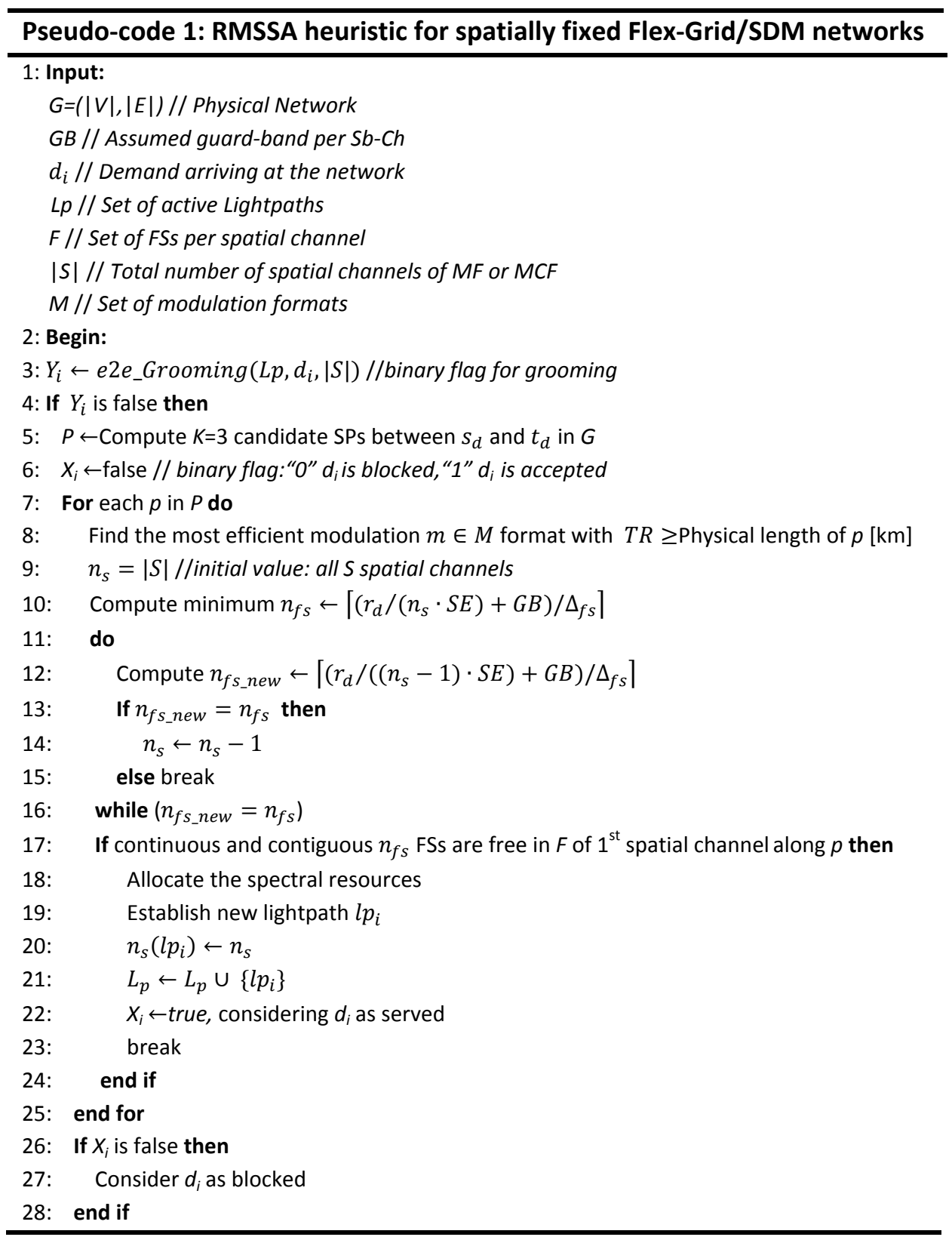




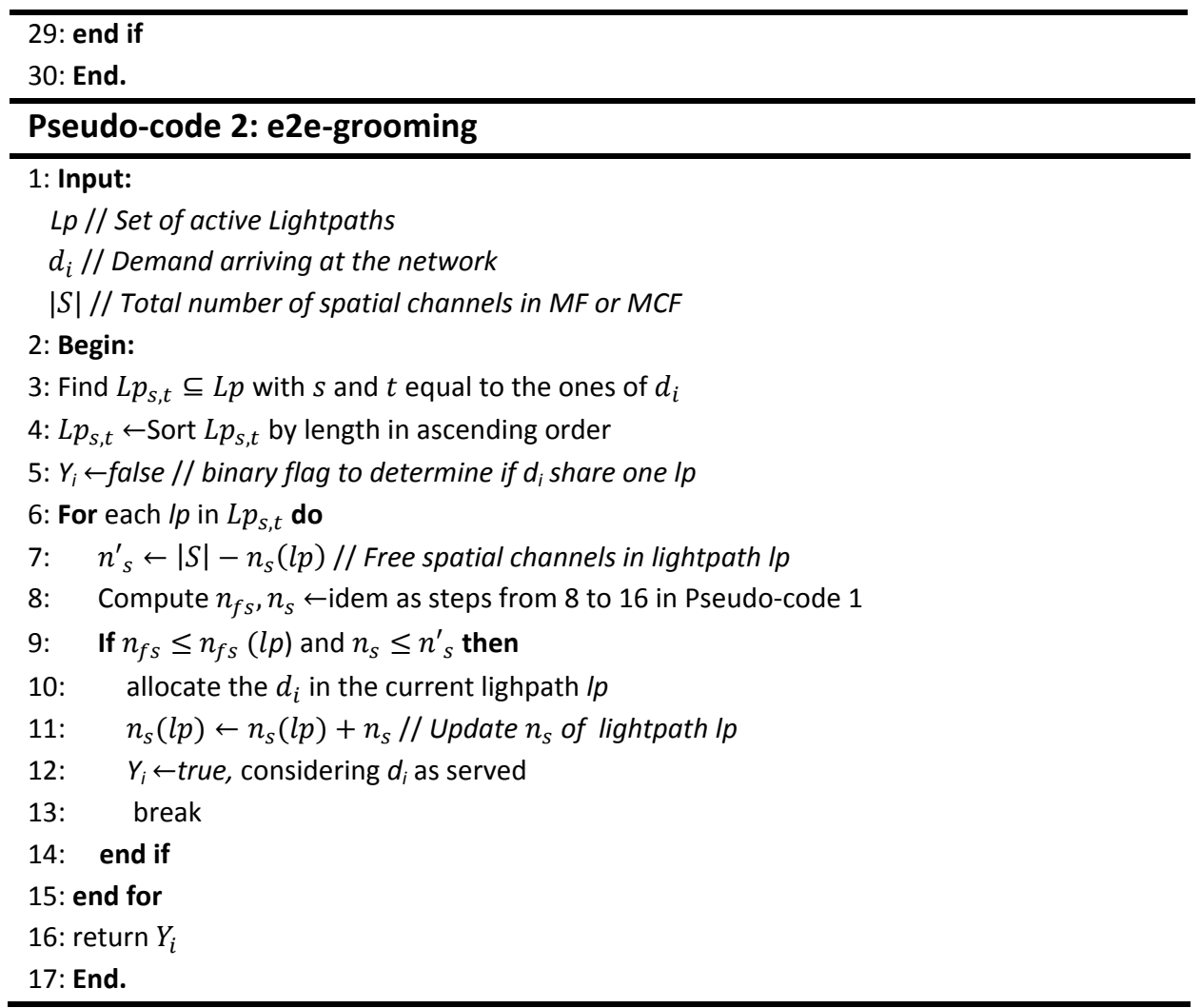

\subsection{SCh allocation in SS-FONs}

In the previous Spa-SCh configuration, the spatial dimension is considered fixed, and if there are Spa-SChs that do not employ the spatial channel count $|S|$, the aggregation of traffic demands with common source-destination nodes can be allowed. This obviously simplifies the ROADM architecture since JoS reduces the number of SSSs; however, these SSSs could require a high port count [20]. In contrast, in order to introduce flexibility in the spatial domain, it is required a more complex switching technique, like InS (w/ or w/o LC support), as introduced in section 1. This flexibility in the spatial domain means that we can accommodate SChs using a flexible number of spatial channels $\left(n_{s}\right)$ and if spatial channels remain unused $\left(n^{\prime}{ }_{S}=|S|-n_{S}\right)$ in the same or different spectrum portion, these resources can be assigned to other traffic demands, regardless of their $s_{d}$ and $t_{d}$ nodes.

Let us take the previous example in Fig. 2 again, where demand $d_{2}$ could not be allocated in the same spectrum portion assigned to demand $d_{1}$ (in spatial channels $s_{4}$ to $s_{7}$ ) due to the restrictions of the JoS technique. However, in SS-FONs, this demand $d_{2}$ is not only allocated over these spectral and spatial resources, but would also use 1 FS (as $d_{2}$ requires) employing another BVT with one OC at a central frequency different than $f_{i}$. Obviously, the complexity and cost of both ROADM and BVT designs enabling spectrally-spatially flexible SCh configurations are higher [16][26].

Depending on the $n_{S}$ value, different SCh allocation policies can be configured, namely, SpeSCh, Spa-SCh or S2-SCh. For instance, if $n_{s}=1$, we have a Spe-SCh [Fig. 1(c)], while a Spa-SCh [Fig. 1(a)] or an S2-SCh [Fig. 1(b)] could be allocated with higher $n_{s}$ values depending on the 
spectral width of each $\mathrm{Sb}-\mathrm{Ch}\left(\Delta_{S b}\right)$. Starting from $n_{s}=1$ (i.e., a Spe-SCh generated with MCs), if $n_{s}$ increases, $n_{f s}$ can start decreasing until only SC may be needed (Spa-SCh).

As we have a wide range of possibilities to form SChs (i.e., different $n_{s}$ versus $n_{f s}$ options) with SS-FONs, it can be useful to choose the proper configuration $\left(n_{s}, n_{f s}\right)$ based on a function $\left(b_{i}\right)$ that weights spectral and spatial resources. This function $b_{i}$ is shown in Eq. (2), where $\alpha \in[0,1]$ is the weighting parameter.

$$
b_{i}=\alpha \cdot n_{f s}+(1-\alpha) \cdot n_{s}
$$

For each demand bit-rate $r_{d}$, given the modulation format to be used and the total spatial channel count $|S|$, we compute the solution space $\Omega$, considering any candidate $\left(n_{s}, n_{f s}\right)$ tuple. To populate it, from $n_{s}=1$ onwards we compute the corresponding $n_{f s}$ by means of Eq. (1). Tuples with identical $n_{f s}$ value, but with higher $n_{s}$ are directly discarded. Subsequently, for a given $\alpha$ value, $b_{i}$ is computed for each tuple and added to set $\beta$. The selected $\Omega$ tuple will be the i-th element yielding lowest $b_{i}$ value. Fig. 3 shows the example of three $\alpha$ values: 0 [Fig. 3(a)], 0.5 [Fig. 3(b)] and 1.0 [Fig. 3(c)] with transceivers of 32 GBaud ( $\max \Delta_{S b}=32 \mathrm{GHz}$ ). We have three demands, $d_{1}=1 \mathrm{~Tb} / \mathrm{s}, d_{2}=100 \mathrm{~Gb} / \mathrm{s}$, and $d_{3}=400 \mathrm{~Gb} / \mathrm{s}$, employing PM-64QAM, PM-16QAM, PM-QPSK, respectively. Given $|S|=7$, the $\Omega$ sets for demand $d_{1}, d_{2}$ and $d_{3}$ are:

$\Omega_{1}=\{(1,8),(2,4),(3,3),(5,2)\}$

$\Omega_{2}=\{(1,2),(3,1)\}$

$\Omega_{3}=\{(1,9),(2,5),(3,4),(4,3),(6,2)\}$

In Fig. 3(a), when $\alpha=0$ the prioritization will be to minimize the number of allocated spatial channels $n_{s}$ per SCh and the selected tuple is the first element in the respective $\Omega$ set, i.e., $\Omega(1)$. Conversely, when $\alpha=1$ [fig. 3(c)] the objective is to minimize the allocated spectral resources, so the selected tuple is the last element in $\Omega$, i.e., $\Omega(|\Omega|)$. However, when $\alpha \in(0,1)$, e.g., $\alpha=0.5$ [fig. 3(b)], $\beta$ sets for demand $d_{1}, d_{2}$ and $d_{3}$ are:

$\beta_{1}=\{4.5,3.0,3.0,3.5\}$,

$\beta_{2}=\{1.5,2.0\}$,

$\beta_{3}=\{5.0,3.5,3.5,3.5,4.0\}$.

In this case, for $\alpha=0.5$ the lowest $b_{i}$ for each demand are shown in boldface and the selected tuple $\Omega(i)$ is different for each $d$. In fact, $\Omega(3)$ is the selected element for $d_{1}, \Omega(1)$ for $d_{2}$, and $\Omega(4)$ for $d_{3}$. This approach implies that a mix of different SCh configurations can be allocated to satisfy the offered traffic.

It can occur that two or more elements in set $\beta$ have the same $b_{i}$ value. In these cases, we select the element requiring the least amount of spectral resources, because we also aim to minimize the number of OCs in the case of MC SChs (Spe-SChs or S2-SChs), due to potential cumulative coherent crosstalk between contiguous $\mathrm{Sb}$-Chs.

The aggregate traffic is equally split into $n_{S}$ spatial channels and $n_{O C}$ OCs $\left(n_{O C}=\left\lceil\frac{r_{d} /\left(n_{s} \cdot S E\right)}{\max \Delta_{S b}}\right\rceil\right)$. In the example, for $d_{1}=1 \mathrm{~Tb} / \mathrm{s}$ PM-64QAM and $\alpha=0.5, n_{s}=3$, the resulting symbol rate is 27.78 GBaud $[1000 \div(3 \cdot 12)]$ and $n_{O C}$ equal to 1 . Therefore, the bit-rate transmitted per each Sb-Ch is $333.33 \mathrm{~Gb} / \mathrm{s}[1000 \div(3 \cdot 1)]$. Note that, when MC SChs have to be allocated, 
e.g., as the ones depicted in Fig. 3(a), and Fig. 3(b), MC BVT with same modulation format are considered, in order to transmit/receive the aggregate bit-rate of the traffic demands [see the simplified BVT diagram in Fig. 1(b,c)].

In Fig. 3(a) for $\alpha=0$, each demand is allocated over one Spe-SCh, namely, SCh1, SCh2 and SCh3 for $d_{1}, d_{2}$ and $d_{3}$, respectively. Each Sb-Ch is modulated over one OC at different operational central frequency (therefore, different laser sources, i.e., one per $\mathrm{OC}$ are required). For example, in Fig. 3(a) we should employ 8 laser sources. As $\alpha$ value increases, the possibility of sharing the laser source between Sb-Chs also increases, thanks to the spatial BVT designs [27]. For instance, with $\alpha=0.5$ and $\alpha=1$, we can observe in Fig. 3(b,c) that Spa-SChs allow to reduce the number of laser sources to 3 . Blanks in these figures are free spectral and spatial resources to be assigned to other demands that fit into them. Moreover, with $\alpha=1$ the total number of Sb-Chs (therefore, transceivers) to allocate SCh1, SCh2, SCh3 are higher than those with $\alpha=0$ and $\alpha=0.5$ (14 vs. 8), whilst the operational symbol rate of the transceivers is lower with $\alpha=1$ than those with $\alpha=0$ and $\alpha=0.5$. For example, 16.67 GBaud vs. 27.78 GBaud for $d_{1}$. It is worth observing that, like in the case of our example for $\alpha=0.5$, it should exist an alpha value where the number of $\mathrm{Sb}$-Chs and the spectral width per each are equal to alpha 0 , with the difference that the Sb-Chs are accommodated over the spatial domain instead of the spectral one.

$\alpha=0$ is the unique value that ensures that a single allocation policy is employed (Spe-SCh) for all demands, regardless of the traffic profile, network size, fiber type and $|S|$. However, a mix of Spe-SCh with the other SCh allocation policies (i.e., Spa-SCh and S2-SCh) can be configured with higher $\alpha$ values, including $\alpha=1$ because $n_{O C}$ depends, among other factors, on the maximum operational symbol rate of the transceivers. Pseudo-code 3 shows the RMSSA greedy heuristic that we propose for spectrally-spatially flexible super-channel configuration. In general, when a new demand $d_{i}$ arrives at the network, $\mathrm{K}=3$ Shortest Paths (SPs) are computed as feasible candidate paths. For each candidate path $(p)$, the most efficient modulation format, with a TR equal or higher than the physical path length, is selected. Then, all candidate configurations for SCh composition $(\Omega)$ are computed by means of Pseudo-code 4 (line 7), which returns the selected tuple $\left(n_{s}, n_{f s}\right)$ based on the alpha parameter. Finally, if there exist $n_{f s}$ FSs available in $n_{s}$ spatial channels along $p$, demand $d_{i}$ is allocated. Otherwise, subsequent candidate paths are analyzed. If no candidate path can allocate $d_{i}$, it is finally blocked.

The analysis of the time complexity of the presented RMSSA heuristic for SS-FONs can be presented as follows. First, the algorithm computes the K-SPs by using Yen's algorithm whose complexity was already mentioned when discussing Pseudo-code 1 overall complexity. Later on, for each $p \in P$ the most efficient modulation format is selected followed by the selection of the SCh configuration over $|S|$ available spatial channels, therefore, the time complexity of this step is again bounded by $|P|(|M|+|S|)$. To this time complexity has to be added the one of searching through all $|P|$ feasible candidate paths (worst-case) free spectral resources in $F$ over up to $|S|$ spatial channels per link $e \in E$. So at the end, the overall time complexity of RMSSA greedy heuristic per arrival connection for SS-FONs is $\mathcal{O}(K|V|(|E|+|V| \cdot \log |V|)+$ $|P|(|M|+|S|+|F||S||E|))$. Compared to the RMSSA heuristic for spatially fixed FlexGrid/SDM networks, the time complexity of this RMSSA heuristic for SS-FONs increases by $|S|$ times in one of its terms, being necessary to search free spectral resources in $F$ not only over 
the first spatial channel but also over up to $|S|$ spatial channels per link. This fact gives us an idea of the complexity increment that the control plane would have to handle during the SCh provisioning tasks in SS-FONs versus those required in fixed Flex-Grid/SDM networks.

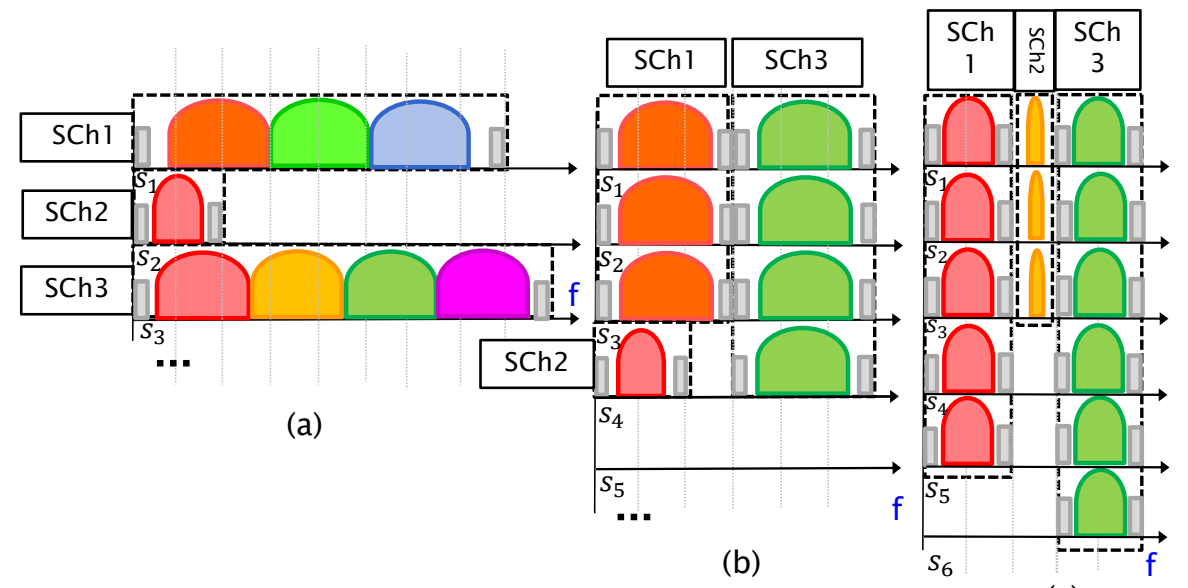

(c)

Fig. 3. SCh allocation for $\mathrm{d}_{1}=1$ Tbps, $\mathrm{d}_{2}=100 \mathrm{Gbps}, \mathrm{d}_{3}=400$ Gbps with PM-64QAM, PM-16QAM, PMQPSK, respectively, $|S|=7$ with: (a) $\alpha=0$, (b) $\alpha=0.5$ and (c) $\alpha=1$.

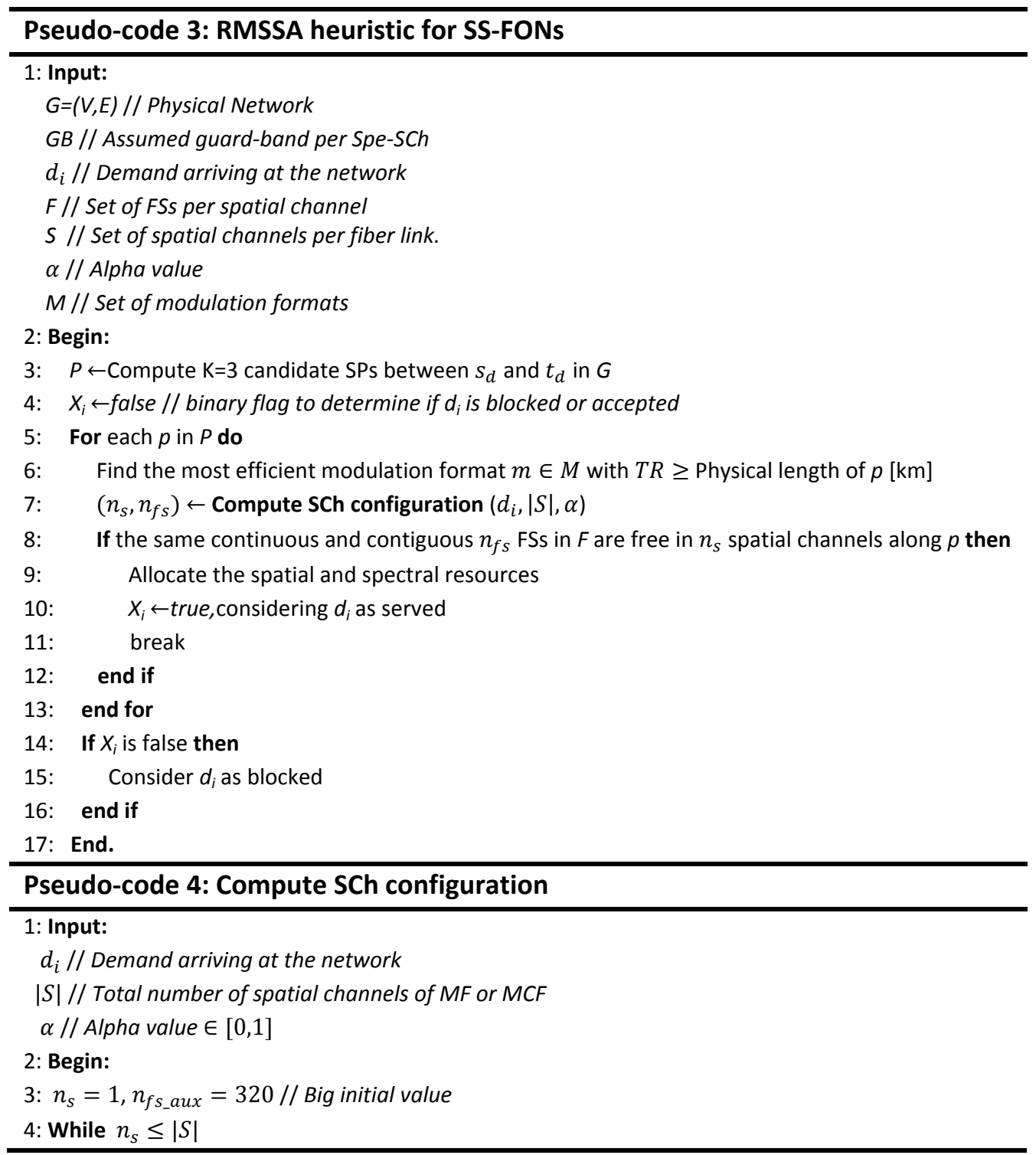




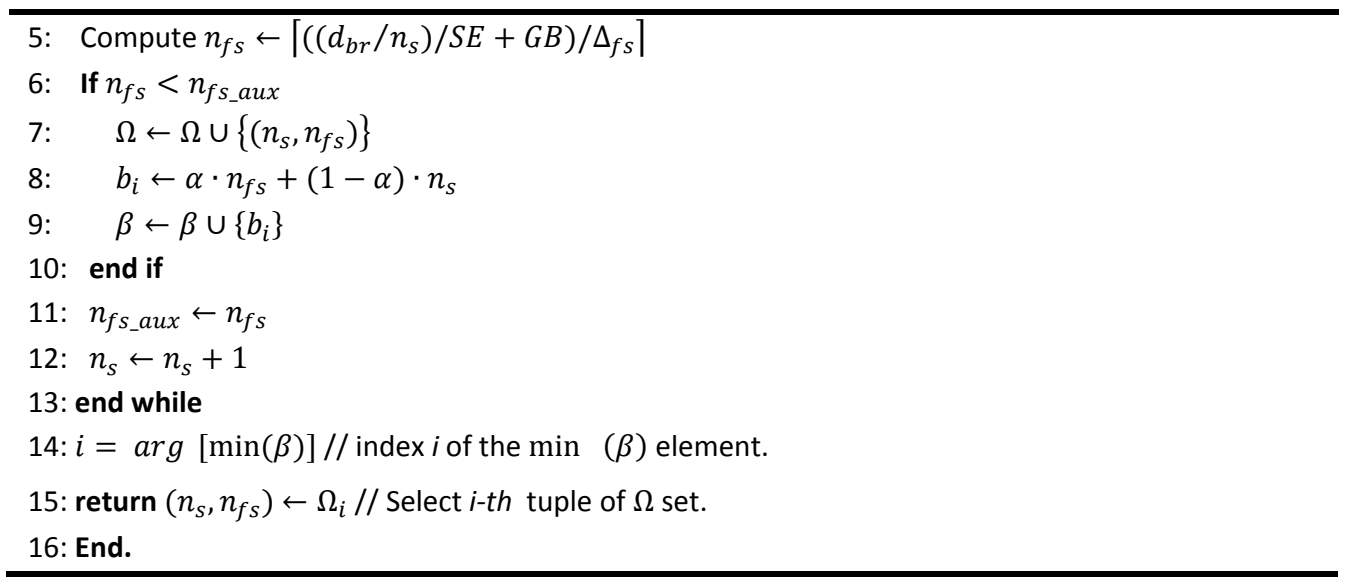

\section{Numerical Results}

\subsection{Scenario Details and Assumptions}

In order to estimate the throughput scalability of Flex-Grid/SDM networks, we consider two reference networks: the Deutsche Telekom National backbone network (DT12) and a continental US backbone network (US26), whose topologies and main characteristics are shown in Fig. 4. The network diameter is defined as the longest shortest path between any pair of nodes. In each network link, we scale up the spatial multiplicity $|S|$ from 7 to 30 . We consider MF- as well as MCF-based solutions. For MF ones, 1-spatial channel granularity is considered, while for MCF ones the five best single-mode MCF prototypes found in the literature [6]-[10] are taken into account, i.e., $|S| \in\{7,12,19,22,30\}$. The worst aggregate ICXT measurements for the considered cases can be seen in Table I. The transmission reach (TR) has been estimated as the most restricted value between the ones limited by SNR and ICXT. For SNR we use the Gaussian-Noise (GN) model [21]. More details about the TR estimations can be found in our previous work [11]. Table II shows the overall TR estimations for each MCF type and modulation format. The values in red represent those situations where TR is limited by ICXT. Note that the 30-core MCF prototype has lower ICXT than 19-core and 22-core MCF prototypes because it is a heterogeneous design. Each fiber/core has 320 available FSs of $12.5 \mathrm{GHz}$ width. We also consider that the GB between SChs is equal to 7.5 $\mathrm{GHz}[11]$.

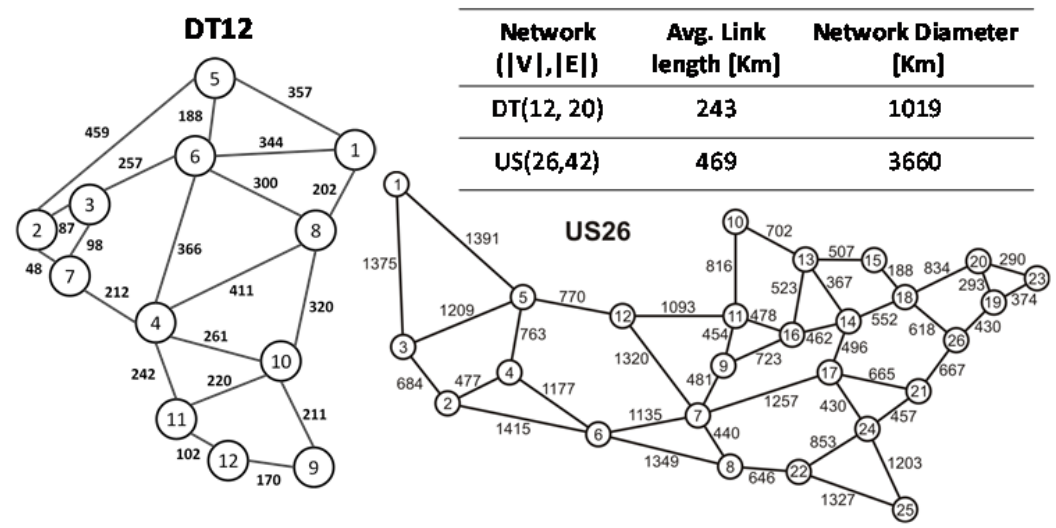

Fig. 4. Considered reference network topologies. 
A dynamic network scenario is assumed, where demand requests arrive at the network following a Poisson process with negative exponentially distributed Inter-Arrival Time (IAT). Each request asks for a bidirectional lightpath between uniformly distributed source and destination nodes with bit-rate equal to $r_{d}$ during a certain Holding Time (HT), which also follows a negative exponential distribution. We consider two traffic profiles (TPS): $\operatorname{TP} 1=\{100$, $400,1000\} \mathrm{Gb} / \mathrm{s}$ with probabilities $\{0.4,0.3,0.3\}$, so that the mean bit-rate is $460 \mathrm{~Gb} / \mathrm{s}$; and TP2 $=\{400,1000,2000\} \mathrm{Gb} / \mathrm{s}$ with the same previous probabilities, resulting in a mean bit-rate equal to $1.06 \mathrm{~Tb} / \mathrm{s}$. Different offered loads (HT/IAT) are simulated until we obtain a Bandwidth Blocking Probability (BBP) close to $1 \%$ for each spatial multiplicity value. To get statistically relevant results, we offer $5 \times 10^{5}$ bidirectional connection requests per execution.

TABLE I

Measured worst aggregate ICXT at 1550nm.

\begin{tabular}{c|c|c|c|c}
\hline \hline $\mathbf{7}$ cores [6] & $\mathbf{1 2}$ cores [7] & $\mathbf{1 9}$ cores [8] & $\mathbf{2 2}$ cores [9] & 30cores[10] \\
\hline$-84.7 \mathrm{~dB} / \mathrm{Km}$ & $-61.9 \mathrm{~dB} / \mathrm{Km}$ & $-54.8 \mathrm{~dB} / \mathrm{Km}$ & $-56.2 \mathrm{~dB} / \mathrm{Km}$ & $-60 \mathrm{~dB} / \mathrm{Km}$ \\
\hline
\end{tabular}

TABLE II

Overall TR Estimation in km.

\begin{tabular}{ccccc}
\hline \hline MCF & BPSK & QPSK & 16-QAM & 64-QAM \\
\hline \hline 7 & $>20000$ & 9000 & 2000 & 600 \\
\hline 12 & $>20000$ & 9000 & 2000 & 600 \\
\hline 19 & 4755 & 2383 & 599 & 150 \\
\hline 22 & 6607 & 3311 & 832 & 209 \\
\hline 30 & 15849 & 7943 & 1995 & 501 \\
\hline
\end{tabular}

\subsection{Network Throughput Scalability of spatially fixed Flex-Grid/SDM networks}

This subsection aims to assess the throughput scalability of spatially fixed Flex-Grid/SDM optical networks. As explained in Subsection 2.2, JoS is the applicable switching strategy when the spatial dimension is considered fixed. The results of this subsection correspond to JoSenabled Flex-Grid/SDM networks with e2e-grooming, as the lower bound of spatially fixed Flex-Grid/SDM networks, mandatory for strongly coupled MCFs or FMFs and the cheapest one solution regarding the bypass part of SDM-ROADMs [20]. To this end, we scale up the spatial multiplicity $|S|$ from 7 to 30 spatial channels and for each point we measure the average carried traffic -hereafter referred to as network throughput (in $\mathrm{Pb} / \mathrm{s}$ )- for a $\mathrm{BBP} \approx 1 \%$. We compute this network throughput as the average of the instantaneous carried traffic samples after each lightpath setup/release.

Fig. 5 shows the network throughput for different $|S|$ values in the DT12 and US26 networks, under both TP1 and TP2. Focusing on the MF solution, increasing $|S|$ does not always imply a reduction of the allocated spectrum, due to the spectral grid constraint. Besides, JoS penalizes spectrum occupation, as unused spatial channels cannot be allocated to other demands. These two factors cause the step-like shape seen in Fig. 5.

In the DT12 network, the results presented in Fig. 5(a,b) disclose that the spatial multiplicity is poorly exploited without e2e-grooming (NonGr plots). As observed, for a $4.3 \mathrm{x}$ spatial multiplicity factor (i.e., from $|S|=7$ to $|S|=30$ ), network throughput scales only up to $1.5 x$ and 2x for TP1 and TP2, respectively. However, when e2e-grooming is considered ( $\mathrm{Gr}$ plots), network throughput increases considerably, around $3.5 \mathrm{x}$ and $3.3 \mathrm{x}$ for TP1 and TP2, 
respectively. As for the US26 network [Fig. 5(c,d)], which has longer network diameter, smaller differences between these scenarios with or without e2e-grooming are observed. The fact is that connections over long distance paths require employing less efficient modulation formats. Then, the transmitted bit-rate per spatial channel is lower and, consequently, higher $n_{s}$ values are needed, which reduces the possibilities of reusing lightpaths. This effect is even more evident with larger demands. For example, under TP2 [Fig. 5(d)], the results with e2egrooming are practically equal (or very close) to those without it, whilst differences are more significant under TP1 [Fig. 5(c)], particularly as the spatial multiplicity increases (e.g., from $|S|=15$ onwards). Moreover, for $4.3 x$ spatial multiplicity factor, network throughput with e2egrooming scales up to a factor very similar as in the DT12 network, namely, about 3.1x and 3.5x under TP1 and TP2, respectively. As highlighted before, the performance of spatially fixed Flex-Grid/SDM networks is sensitive to the network size and TP. For example, in the DT12 network, Fig. 5(a,b), the e2e-grooming effect is evidenced earlier under TP1 (from $|S|=8$ onward) than under TP2 (from $|S|=15$ onward). In contrast, in the US26 network, the e2egrooming effect is evidenced from $|S|=14$ onward under TP1, while almost inappreciable under TP2. The higher the network size (less efficient modulation formats have to be explored) or the bigger the $r_{d}$ value, the larger the allocated $n_{s}$ leaving less spatial resources for e2e-grooming.
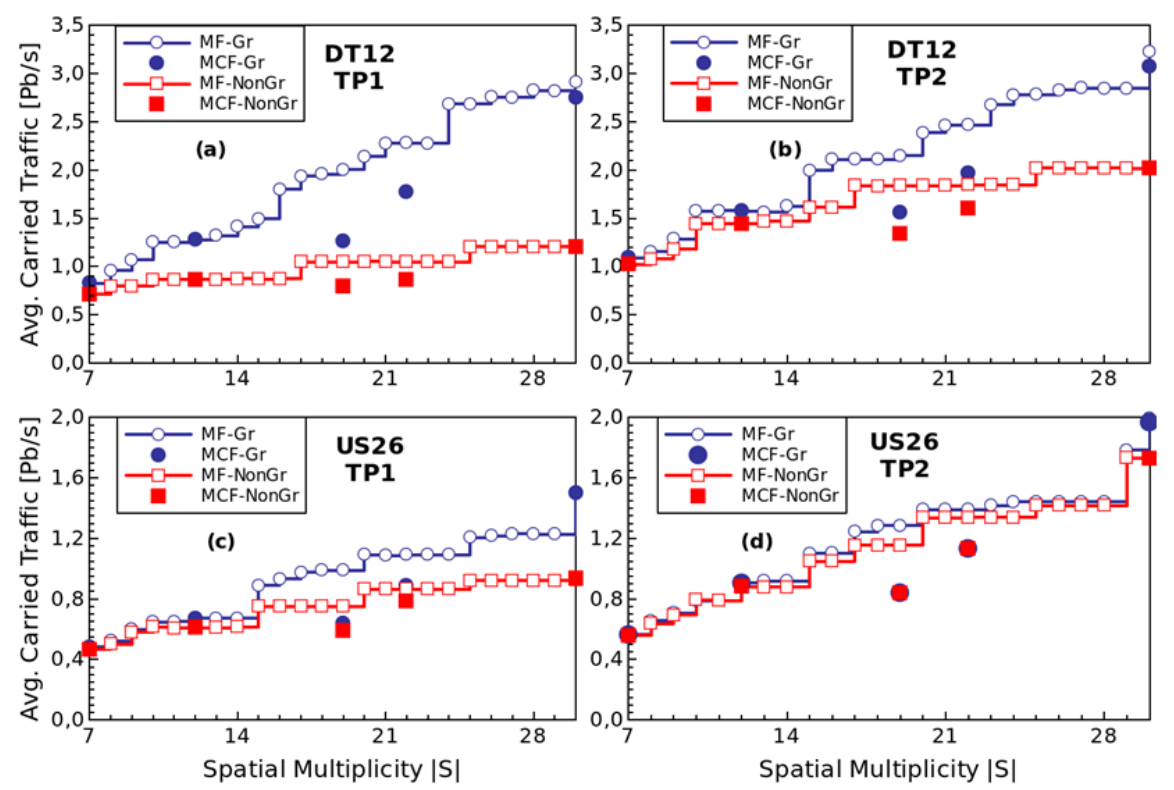

Fig. 5. Network Throughput (in $\mathrm{Pb} / \mathrm{s}$ ) versus $|\mathrm{S}|$ in JoS-enabled Flex-Grid/SDM networks for: (a) DT12 under TP1, (b) DT12 under TP2, (c) US26 under TP1, (d) US26 under TP2.

Regarding MCFs, as expected, the larger the network size, the higher the ICXT impact. ICXT forces to employ more robust modulation formats along a physical path, and may increase both spectral and spatial allocated resources. In both DT12 and US26 networks, 7-core, 12core and 30-core MCFs evidence the same or very close performance as the MF equivalent solutions, since their extremely low ICXT does not become the TR limiting factor, being SNR instead, as in the equivalent MF solutions. However, the 19- and 22-core MCFs exhibit substantial differences against the equivalent MF solutions. Since the 19-core MCF shows the highest ICXT level, network performance degradation is relevant. Indeed, these differences in terms of network throughput are up to 50\% (in the worst cases) for both DT12 and US26 network. Regarding the e2e-grooming utilization, significant benefits are obtained especially in 
the DT12 network [Fig. 5(a,b)], whilst in the US26 network [Fig. 5(c,d)] ICXT (for 19-core and 22-core MCFs) may prevent any lightpath reuse. Finally, the network throughput for MCF scales up to practically the same factor as the MF solution ( $0 \%$ penalty versus MF solution, except for penalties evidenced in the 19- and 22-core MCFs) in both DT12 and US26 networks, which indicates the feasibility of MCF deployments in the assessed spatial multiplicity interval.

\subsection{Network Throughput Scalability of SS-FONs}

In this subsection, we aim to discuss how different SCh configurations influence network throughput of SS-FONs. We also contrast the network throughput scalability versus hardware requirements in the transponder design. For these purposes, InS with LC change support strategy is assumed at the network nodes because it allows reaching the maximum performance of SS-FONs [14].

\subsubsection{SCh configuration Effect in Network Throughput and Transponder Complexity}

As explained in subsection 2.1, different candidate super-channel configurations $\left(n_{s}, n_{f s}\right)$ can be obtained depending on the $\alpha \in[0,1]$ value. In order to illustrate the effect of the $\alpha$ parameter, we choose a network scenario where both MF and equivalent MCF solutions can be contrasted. Both 7- and 12-core MCF prototypes have the same TR as the equivalent MF solutions (as presented in Table II). So, ICXT is not the TR limiting factor. In contrast, the 19core MCF prototype evidences lower TR than the equivalent MF solution. Moreover, this prototype shows the most restrictive TR compared to the 22-core and 30-core MCF ones. Hence, we find interesting to evaluate the $\alpha$ effect in both reference networks, considering all links equipped with 19-core MCFs [8]. Fig. 6(a,b) show the network throughput (for a BBP $\approx 1 \%$ ) versus $\alpha \in[0,1]$, with 0.02 granularity, in DT12 and US26 networks, respectively. The mean bit-rate per connection is 800 Gbps.

Please recall that $\alpha=0$ forces allocating all demands over Spe-SChs, where the overhead due to $\mathrm{GBs}$ is lower than in other SCh configurations. This aspect may suggest that the highest network throughput is always obtained with $\alpha=0$, describing a monotonically decreasing behavior from then on. However, observing Fig. $6(a, b)$, we see that network throughput experiences short increments or becomes constant with some high $\alpha$ value intervals. This means that, even though other SCh configurations $\left(n_{s}, n_{f s}\right)$ introduce higher GB overhead, they could be appropriate (especially in dynamic scenarios where fragmentation plays an important role) for increasing the number of allocated connections, thus improving network throughput. 

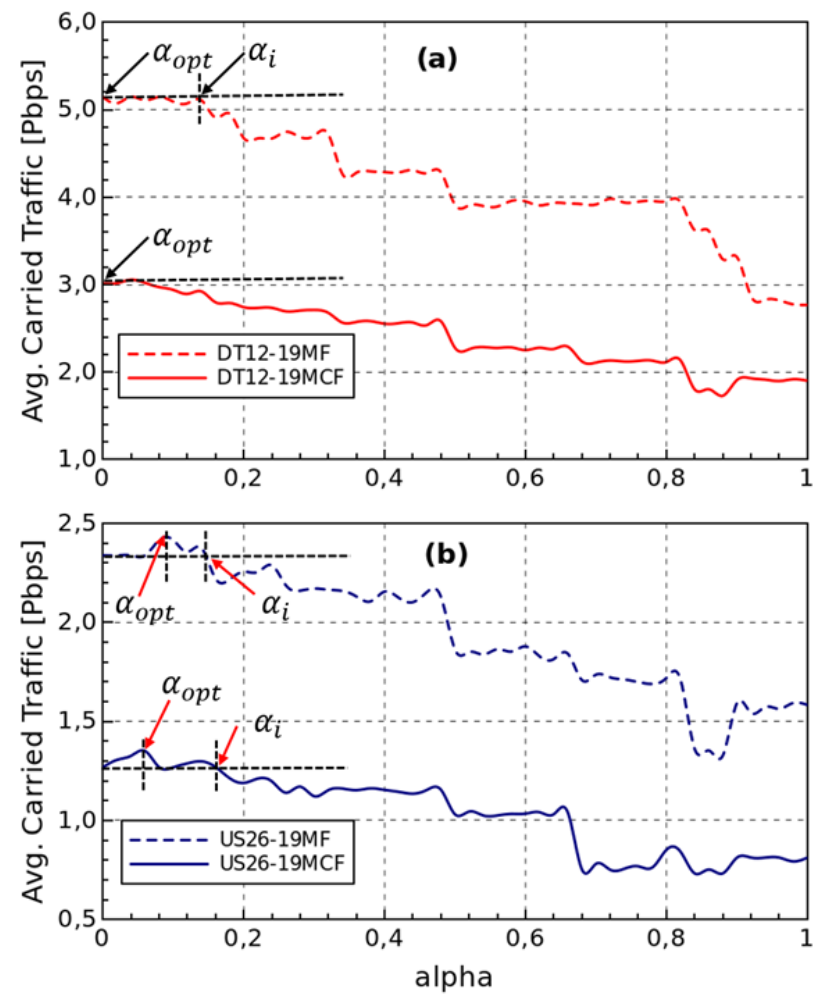

Fig. 6. Network throughput versus $\alpha$ with $|\mathrm{S}|=19$ MF\&MCF solutions in: (a) DT12 network, and (b) US26 network.

The above-described situation can be more relevant in cases where demands require a large number of FSs using a single spatial channel, hindering their allocation along the routing path, as both spectral continuity and contiguity constraints must be ensured end-to-end. Consequently, it may be easier to find less spectral resources, although employing more than one spatial channel (i.e., using Spa-SChs or S2-SChs). In MCF-enabled networks, we also have to recall that the higher the ICXT (and the larger the network size), the higher the amount of spectral resources to be allocated, as less efficient modulation formats are mandatory. This is evidenced in Fig. 6(b) for both 19MF (dotted blue line) and 19MCF (solid blue line) solutions, where $\alpha=0.08$ and $\alpha=0.06$ allows increasing the network throughput compared to $\alpha=0$, namely, $2.42 \mathrm{Pbps}$ vs. $2.34 \mathrm{Pbps}$ (throughput gain $=3.4 \%$ ) and $1.35 \mathrm{Pbps}$ vs. $1.27 \mathrm{Pbps}$ (throughput gain $=6.3 \%$ ), respectively. These $\alpha$ values yielding the highest network throughput we denote them as optimal values $\left(\alpha_{\text {opt }}\right)$, as highlighted in Fig. 6 . Meanwhile, in the scenarios where the network diameter is not significant (like in DT12 network) the initial alpha equal to 0 can lead to the maximum throughput (i.e., $\alpha_{o p t}=0$ ). We can also see in this figure that the network throughput vs. alpha function is not linear, but rather like a step function. There are intervals where no significant changes occur when incrementing $\alpha$, and some points where the network throughput drops abruptly. Another interesting point from Fig. 6 to be observed is the one denoted in this work as the break-even point $\left(\alpha_{i}\right)$, which is defined as the maximum alpha value after $\alpha_{\text {opt }}$ that yields identical network throughput to $\alpha=0$ (i.e., throughput gain $=0 \%$ ). This point is also highlighted in Fig. $6(a, b)$. The situations where network throughput is not affected by $\alpha$ increments can offer benefits in the BVT complexity (and therefore in cost), which will be discussed later on. According to the results, throughput reductions up to $48 \%$ and $38 \%$ are reported for MF and MCF solutions, respectively, when comparing $\alpha=0$ vs. $\alpha=1$. Finally, comparing MCF versus MF solutions, 
we can see that in the DT12 network with $\alpha=0$, throughput differences become $2.1 \mathrm{Pbps}$ (42\% penalty), whilst for $\alpha=1$ they lower to 0.9 Pbps (33\% penalty). In the case of the US26 network with $\alpha=0$, these differences are around $1.1 \mathrm{Pbps}$ (46\% penalty), while with $\alpha=1$ they are around $0.8 \mathrm{Pbps}$ (50\% penalty).

Another aspect to study is how the network throughput gain ( $\alpha_{\text {opt }}$ vs. $\alpha=0$ ) is affected by the traffic profile. To shed light on this, Fig. 7 shows the throughput gain (\%) for different average bit-rates per connection $(r)$, namely, $0.4 \mathrm{Tbps}, 2 \mathrm{Tbps}$ and $4 \mathrm{Tbps}$, in both reference topologies. We can see that, in the US26 network there are throughput gains for both MCF and MF solutions and for the three traffic profiles. These gains rise as the mean bit-rate per connection increases, becoming up to $40 \%$ in the MCF scenario under $r=4 \mathrm{Tbps}$. That is, for long-haul backbone networks with ICXT-limited TR, the higher the $r$ value, the higher the throughput gain obtained by using the $\alpha_{\text {opt }}$. This happens because, as we stated before, large demands can motivate the exploration of SCh configurations with $n_{s}>1$, in order to find out alternatives to accommodate them. Moreover, the higher the $n_{s}$ value, the higher the laser source sharing factor, as explained in subsection 2.2.

In Fig. 7, spaces where no bars are depicted mean that the throughput gain is null (0\%), i.e., $\alpha_{\text {opt }}=0$. For example, in the DT12 network under $r=0.4$ Tbps and $r=2$ Tbps, $\alpha=0$ is the optimal value for both MF and equivalent MCF solutions. For $r=4$ Tbps, the MCF solution with $\alpha_{\text {opt }}=0.03$ reports a $7 \%$ of throughput gain, whilst the MF solution does not evidence any gain. Although in the MF solution $\alpha_{\text {opt }}>0$ does not occur, the benefits of replacing some Spe-SChs by Spa-SChs by increasing alpha should be analyzed. For example, in this case we obtain by simulation that $\alpha=0.08$ yields identical network throughput as $\alpha=0$ (i.e., $\alpha_{i}=$ 0.08). According to [26], the total SDM network cost can be considered as the sum of the ROADMs cost and BVTs cost. The ROADM cost can be disaggregated in optical express (OE) cost plus Add/Drop module cost and plus amplifiers cost. Among these network elements, BVTs contribute to the total network cost in the range from $45 \%$ to $58 \%$, i.e., BVTs are the major network equipment cost. It has been reported that the use Spa-SCh BVTs can reduce the cost in 5-20\% versus Spe-SCh BVTs with 2-10 Sb-Chs, thanks to the integration and sharing of network elements (e.g., the laser sources). Moreover, for Spa-SChs, the joint digital signal processing [29] of spatial sub-channels at the receivers by means of integrated chips could also potentially reduce the power consumption and cost of the SDM networks. Therefore, using higher $\alpha$ values in dynamic scenarios may not only increase the network throughput, but also reduce the overall SDM network cost. Some statistics about BVT configuration with different alpha values corroborating the last affirmation are presented in the results of the next subsection 3.3.2. 


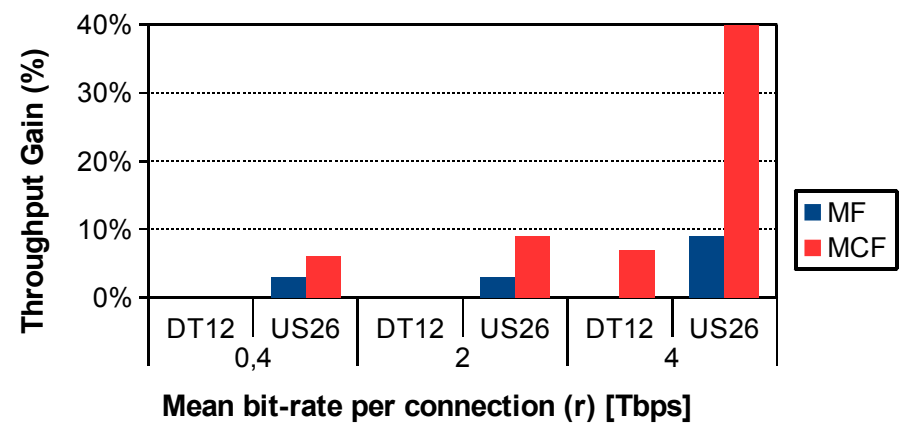

Fig. 7. Throughput gain (\%) for $\alpha_{\text {opt }}$ vs. $\alpha=0$ in DT12 and US26 networks with 19-core MCFs.

To end this subsection, we illustrate in Fig. 8 the different SCh configurations that can be obtained with $\alpha_{\text {opt }}$, showing the $n_{s}$ histogram. For the US26 backbone network [Fig. 8(a-c)], we consider the MCF solution and all $r$ values assumed before, whilst for the DT12 network in Fig. 8(d), we contemplate the MF solution and $r=4$ Tbps. As mentioned before, this latter scenario is an interesting case where no traffic gain exists; however, alternative SCh configurations than Spe-SCh can potentially reduce overall network cost.
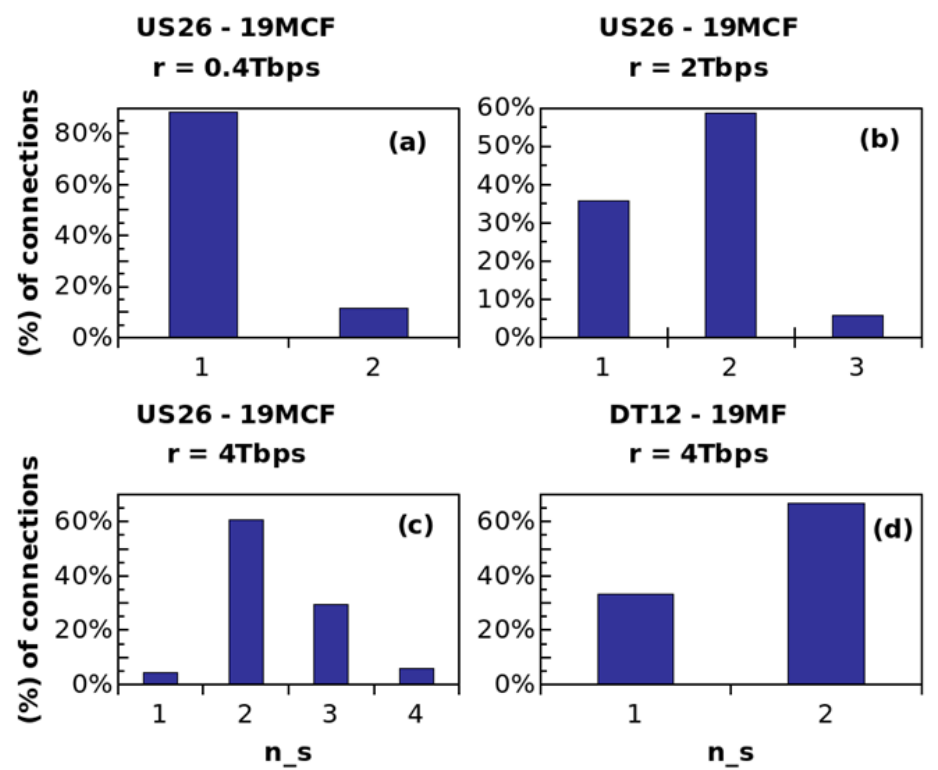

Fig. 8. $n_{s}$ Histogram with $|\mathrm{S}|=19$ (MCF), $\alpha_{\text {opt }}$, US26 for: (a) $\mathrm{r}=0.4 \mathrm{Tbps}$, (b) r=2 Tbps, (c) r=4 Tbps; and (d) $|\mathrm{S}|=19(\mathrm{MF}), \alpha_{i}, \mathrm{DT} 12, \mathrm{r}=4$ Tbps.

As observed, higher $r$ values demand increasing $n_{s}$ as well. In the US26 network, under $r=0.4$ Tbps the $\sim 90 \%$ of connections employ $n_{s}=1$, whilst for $r=2$ Tbps the percentage of connection with $n_{s}=1$ downs to $\sim 35 \%$. Finally, for $r=4$ Tbps just $\sim 5 \%$ of connections employ $n_{s}=1$. As $r$ increases from 0.4 to 4 Tbps, the maximum $n_{s}$ goes from 2 to 4 . These results highlight that a considerable number of Spe-SChs can be replaced by Spa-SChs (SC or MC) with throughput gains and with cost reduction implications, as stated before. Moreover, the previous described scenario in Fig. 7 for DT12 with MF and $r=4$ Tbps, reported that, while there is no $\alpha>0$ that provides throughput gain, there exists an $\alpha_{i}$ value $\left(\alpha_{i}=0.08\right)$ that yields cost reduction in BVTs, keeping the same network throughput as $\alpha=0$. In such case, $\sim 33 \%$ of connections have been allocated with Spe-SChs and the remaining ones employ Spa-SChs with $n_{s}=2$, as appreciated in Fig. 8(d). 


\subsubsection{Throughput vs. Transponder Complexity Analysis}

In this subsection, we analyze the network throughput (for a BBP $\approx 1 \%$ ) of SS-FONs for different SCh configurations in contrast with their associated BVT complexity. To this end, we scale up the spatial multiplicity from 7 to 30 spatial channels, in the two considered networks and under the two TPs introduced in subsection 3.2. Fig. 9 also includes the results of MF solution and the cases with an equivalent MCF prototype. For comparison purposes, network throughput of the JoS-enabled Flex-Grid/SDM networks with e2e-grooming (JoS-Gr plots) previously evaluated in subsection 3.2 is also added in Fig. 9. Moreover, for SS-FONs, we depict the throughput for three alpha values, namely, $\alpha=0, \alpha=1$ and $\alpha_{\text {opt }}$ (the value between 0 and 1 , which produces the highest network throughput).

The ROADM design (OE, Amplifiers and Add/Drop module) for SS-FONs is the same regardless of the $\alpha$ value. However, as we stated in previous section, the selected $\alpha$ value has to be associated with the corresponding complexity and cost of the BVTs. For example, $\alpha=0$ (blue curve with triangle markers) in the DT12 network [Fig. 9(a,b)] yields the highest network throughput, but at expenses of higher complexity and cost derived from using Spe-SCh BVTs. Conversely, $\alpha=1$ (red curve depicted with square markers) yields the lowest network throughput, but with low complexity and cost due to integrated design of the Spa-SCh BVTs [26], [27]. In the case of the US26 network [Fig. 9(c,d)], $\alpha_{\text {opt }}$ (red curve with triangle markers) not only yields higher network throughput than the one with $\alpha=0$, but also lower complexity and cost by replacing some Spe-SChs (e.g., $~ 12 \%$ of connections for US26 backbone network, TP2 and $|S|=19)$ by Spa-SChs or S2-SChs.

Furthermore, for DT12 network, MF/MCF solutions, and TP1/TP2 the results show that $\alpha_{\text {opt }}$ is equal to $\alpha=0$, because higher-order modulation formats allow reducing the spectrum occupation in a single spatial channel. Meanwhile, in the US26 network, $\alpha_{\text {opt }}$ is higher than 0 , particularly, $\alpha_{\text {opt }} \in(0,0.1]$. Throughput gain ( $\alpha_{\text {opt }}$ vs. $\alpha=0$ ) raises as the spatial multiplicity and TP increase (e.g., we demonstrated in previous subsection 3.2.2 up to $40 \%$ of traffic gain with high TPs). Particularly, under TP1, throughput gain keeps lower than $2 \%$, while under TP2, throughput gain raises up to $6 \%$. Throughput gain dependence on the spatial multiplicity can be explained as follows. The smaller the spatial multiplicity, the higher the relative overhead. As the spatial multiplicity increases, the impact of the overhead becomes compensated by the increment of the potential network capacity in terms of spectrum. We also observe that for both considered networks, regardless of the TP, the curves for SS-FON with $\alpha_{\text {opt }}$ and $\alpha=1$ are divergent. They start (in $|S|=7$ ) with a short difference in terms of network throughput (lower than $50 \%$ ), whilst they end (in $|S|=30$ ) with a difference up to $130 \%$. 

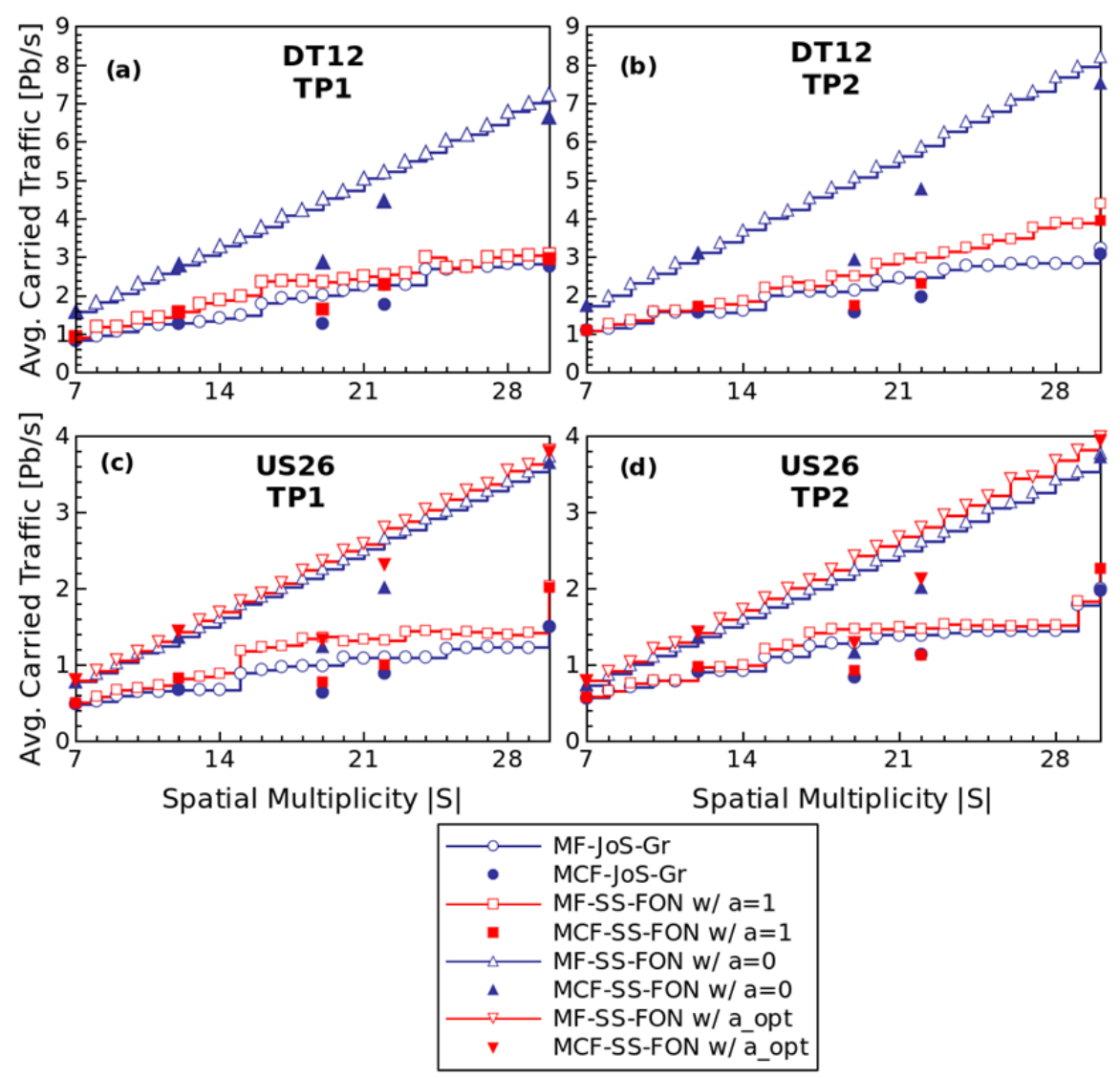

Fig. 9. Network Throughput (in $\mathrm{Pb} / \mathrm{s}$ ) versus $|\mathrm{S}|$ with spectrally-spatially flexible SChs for: (a) DT12 under TP1, (b) DT12 under TP2, (c) US26 under TP1, (d) US26 under TP2.

Regarding MCF solutions, it is important to recall that ICXT may cause a huge amount of required spectral resources per $\mathrm{SCh}$, being necessary to split the optical signals in more spatial channels. In these cases, the $\alpha$ granularity is relevant to find out the optimal value as we have more elements in the solution space $(\Omega)$. For example, for 19-MF $\alpha_{o p t}=0.09$, while for 19core MCF $\alpha_{o p t}=0.04$. Moreover, network throughput with 19-core MCFs is lower or equal than with 12-core MCF due to the higher ICXT, meaning that although 19-core MCFs offer more capacity, the spectral and spatial resources are rapidly consumed by traffic demands that employ less efficient modulation formats than the ones used with 12-core MCFs.

The results of Fig. 9 also report that in SS-FONs for a $4.3 x$ spatial multiplicity factor $(|S|=30$ vs. $|S|=7$ ) with $\alpha=0$ or $\alpha_{\text {opt }}$, throughput scales up to a similar factor (e.g., $5 \mathrm{x}$ and $4.1 \mathrm{x}$ in the best US26 TP2- and worst case -DT12 TP1-, respectively) for MF and MCF solutions, showing a more clear correspondence with the potential network capacity increment given by SDM. Moreover, the network throughput of MCF solution scales up to a close factor to MF one (penalty lower than $10 \%$ against MF solution) in both national and continental backbone networks, which indicates the feasibility of MCF deployments in the assessed spatial multiplicity interval. In the case of $\alpha=1$, this alpha value implies the same complexity and cost in BVTs for spatially fixed Flex-Grid/SDM networks and SS-FONs. Under this scenario, differences (in terms of throughput) between both networks are at most $52 \%$ and even $0 \%$ in some cases. That is, spatially fixed Flex-Grid/SDM networks can carry an acceptable amount of throughput compared to SS-FONs (when $\alpha=1$ ) with the advantage of cost reduction in the ROADM design. It is worth mentioning that, for spatially fixed Flex-Grid/MCF networks end-to- 
end MIMO compensation can be applied to entire spatial domain, while for MCF-enabled SSFONs MIMO cannot be applied. This additional advantage of spatially fixed Flex-Grid/MCF networks can reduce the differences against MCF-enabled SS-FONs for the same BVT complexity (i.e., the same SCh configuration, $\alpha=1$ ) or even for different configurations. For example, note that for 19-core MCF, MIMO equalization in spatially fixed Flex-Grid/MCF networks would yield a throughput higher than the one of MCF-enabled SS-FONs with $\alpha=1$, and the differences with equivalent MF-enabled SS-FONs would be shorter (penalties lower than $25 \%$ ).

So far, Fig. 9 showed the performance of SS-FONs in terms of throughput while evaluating the spatial multiplicity scalability. Besides, it is also interesting to observe their performance in terms of BBP when increasing the offered load (i.e., fixing the IAT and increasing the HT). To this end, Fig. 10 shows the BBP vs. Offered Load (in Pb/s) for some relevant scenarios. In particular, we consider for this analysis the US26 network (where ICXT effects are more relevant), $|S|=12$ and $|S|=22$ (some intermediate spatial multiplicities where there exists a MCF prototype), traffic profile TP2 (which has shown throughput gains for the considered 730x spatial multiplicity range). Fig. 10(a,b) show the results for SCh configurations with $\alpha=0$ and $\alpha_{\text {opt }}$, while Fig. 10(c,d) show them for $\alpha=1$. According to the results, when MF-based solutions are considered, throughput gains $\left(\alpha_{\text {opt }}\right.$ vs. $\left.\alpha=0\right)$ up to $8 \%$ are obtained for low offered loads yielding 0.01\% BBP [Fig. 10(b)]. However, under offered loads yielding a BBP higher than $1 \%$ and lower or equal to $10 \%$, throughput gains are almost inappreciable. Regarding MCF-based solutions, as ICXT of the 12-core MCF has no impact on the TR, its performance is identical to 12-MF equivalent solution, whereas for the 22-core MCF throughput gains up to $10 \%$ are obtained for a $0.01 \%$ target BBP. Fig. 10 (c,d) show the same performance analysis for SCh configurations with $\alpha=1$. In this case, JoS-Gr plots are also added, and as observed, for MF-based solutions the performance differences between SS-FONs and spatially fixed Flex-Grid/SDM networks are lower than 14\%. Meanwhile, when ICXT is relevant (the case of 22-core MCF) the performance differences between both networks are almost inappreciable (i.e., these performance differences, when ICXT is relevant, can be lowered down to $0 \%$ ). This is due to the long distance paths, high bit-rate connections and ICXT impact, where the unused spectral-spatial resources left by lightpaths cannot be assigned to other traffic demands in spite of the spatial flexibility, unless traffic demands are accordingly split into several chunks. Finally, Fig. 10 clearly shows the differences in the supported offered load between SCh configuration with $\alpha=0, \alpha_{\text {opt }}$ and $\alpha=1$. For example, for a maximum 10\% BBP, networks allocating SChs with $\alpha=0$ or $\alpha_{\text {opt }}$ can support up to $2.2 \mathrm{x}$ more offered load than when they allocate SChs with $\alpha=1$, due to spectrum savings of GBs. 

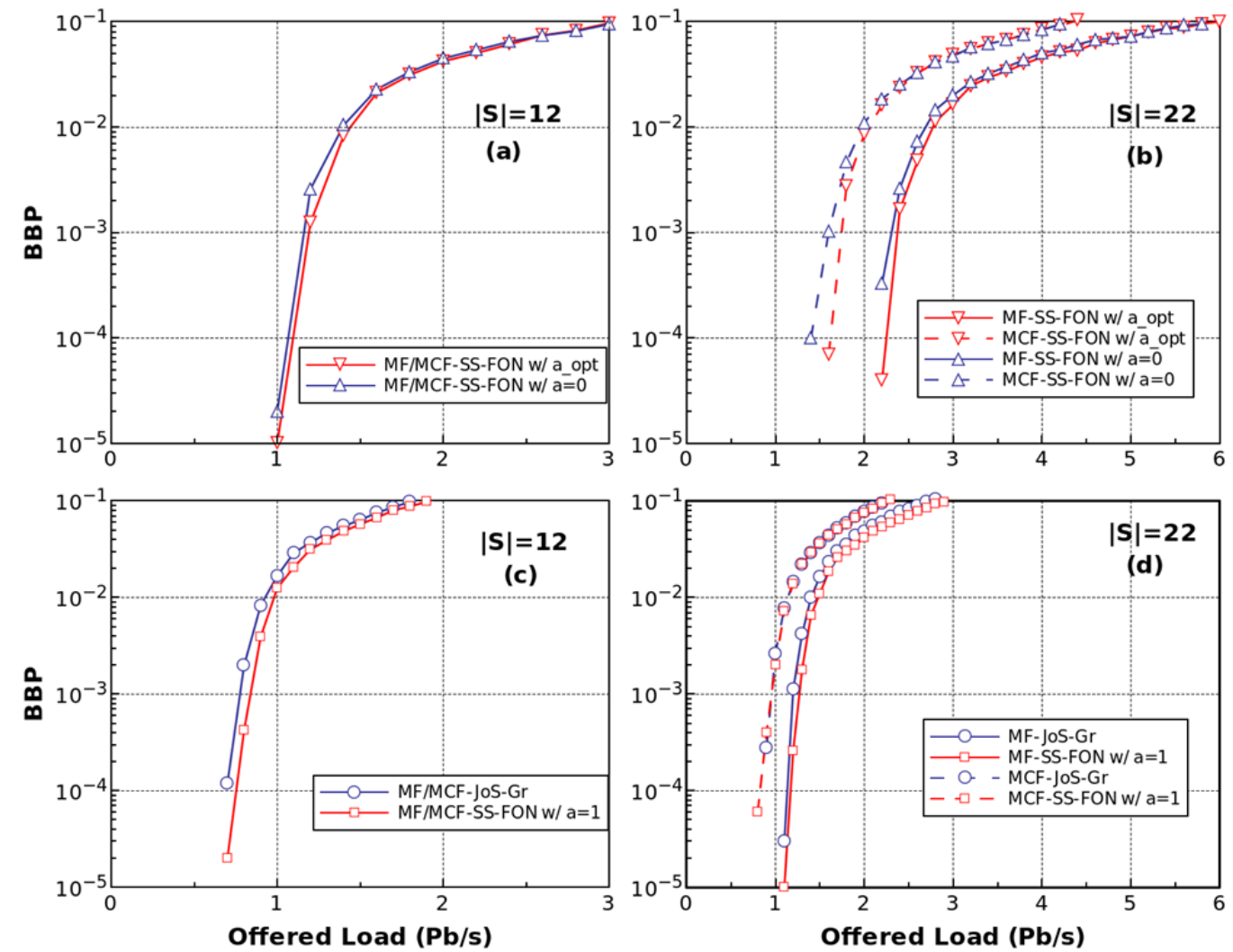

Fig. 10. BBP versus Offered Load (in Pb/s) for US26 network and TP2: (a) $\alpha=0, \alpha_{\text {opt }},|S|=12$, (b) $\alpha=0, \alpha_{\text {opt }},|S|=22$, (c) $\alpha=1,|S|=12$ and (d) $\alpha=1,|S|=22$

Regarding the BVT complexity analysis, we have obtained additional metrics, namely, the average number of OCs per SCh $\left(\overline{n_{O C}}\right)$, equal to the average number of required laser sources per BVT, the average number of transceivers per SCh/BVT $\left(\bar{n}_{T R x}\right)$ and average symbol rate per transceiver $\left(R_{S}\right)$. This last metric can be taken as a key indicator of the electronic complexity of the Digital Signal Processing (DSP). Table III and Table IV show these metrics for both DT12 and US26 networks, under TP2 and with 19-MF and the 19-MCF equivalent solution, respectively. In addition, we consider the SCh configurations ( $\alpha$ values) for JoS-Gr and SS-FONs plots shown in Fig. 9. For both reference networks, regardless of the SDM fiber type, the results show that with $\alpha=0$ we have BVTs with the largest number of OCs and equal to the number of transceivers per BVT. Conversely, when $\alpha=1$, BVTs have only one OC (except for US26 with 19-core MCFs, as there exist cases where 2 Tbps demands with long-distance paths require 2 OCs). That is, Spe-SCh BVTs $(\alpha=0)$ require in average up to $5.8 \mathrm{x}$ and 9.6x more laser sources than Spa-SCh BVTs $(\alpha=1)$ for 19-MCF\&DT12 and 19-MCF\&US26 networks, respectively. In contrast, Spa-SCh BVTs $(\alpha=1)$ require in average up to $2.9 \mathrm{x}$ and $2.1 \mathrm{x}$ more transceivers than Spe-SCh BVTs $(\alpha=0)$. However, when Spa-SCh BVTs $(\alpha=1)$ the large number of transceivers are operating at lower symbol rate than Spe-SCh BVTs $(\alpha=0)$, e.g., up to $62 \%$ and $51 \%$ of symbol rate reduction with $\alpha=1$ against $\alpha=0$ for DT12 and US26 networks, respectively.

This latter aspect supports even more the fact that Spa-SCh BVTs reduces the cost by not only optical integration but also by reducing the electronic processing at DSPs. Therefore, lower amounts of throughput with higher alpha values (see Fig. 6) must be contrasted with cost reduction. Moreover, for $\alpha_{\text {opt }} 4-11 \%$ less laser sources and 2-4\% less transceivers are required per BVT with the same symbol rate, compared to the obtained metrics with $\alpha=0$. As we 
stated before, for DT12 network $\alpha_{\text {opt }}$ is equal to zero, regardless of the SDM fiber type. So, the values for $\alpha=0$ are not repeated in the corresponding column. Table III and IV also show BVT statistics with the previous introduced $\alpha_{i}$ value for US26 network. These results help better understanding the benefits of the proposed mechanism to configure SChs. A 30-32\% less laser sources and 6-9\% less transceivers per BVT with $\alpha_{i}$ yields the same network performance (in terms of throughput) with $\alpha=0$. For DT12 network, this $\alpha_{i}$ value yields the same BVT statistics than $\alpha=0$, then the column for $\alpha_{i}$ in DT12 network is not shown.

Finally, when longer network sizes are considered (e.g., US26 vs. DT12) and lower transmission reaches are obtained (e.g., MCF solution vs. MF one), less efficient modulation format are required to be employed. In turn, less efficient modulation formats determine to transmit lower bit-rate per $\mathrm{Sb}$-Ch. Therefore, in order to satisfy one traffic demand more Sb-Chs (consequently more transceivers, symbol rates and laser-sources) may be required. This is the case in the results of Table III and IV when we compared the different metrics for US26 vs. DT12 networks and for MCF vs. MF solutions.

TABLE III

Metrics for Different SCh Configurations for DT12 and US26 Networks with $|S|=19$ (MF) and TP2

\begin{tabular}{|c|c|c|c|c|c|c|c|c|}
\hline \multirow{3}{*}{ Metric } & \multicolumn{2}{|c|}{ JoS-Gr } & \multicolumn{7}{|c|}{ SS-FON } \\
\cline { 2 - 9 } & \multicolumn{2}{|c|}{$\alpha=1$} & \multicolumn{2}{|c|}{$\alpha=0$} & \multicolumn{2}{|c|}{$\alpha=1$} & $\alpha_{\text {opt }}=0.09$ & $\alpha_{i}$ \\
\cline { 2 - 9 } & DT12 & US26 & DT12 & US26 & DT12 & US26 & US26 & US26 \\
\hline$\overline{n_{O C}}$ & 1 & 1 & 3.8 & 5.4 & 1 & 1 & 4.8 & 3.8 \\
\hline$\overline{n_{T R x}}$ & 10.9 & 11.2 & 3.8 & 5.4 & 10.9 & 11.2 & 5.2 & 5.1 \\
\hline$R_{S}$ & 9.5 & 13.7 & 25.2 & 28 & 9.5 & 13.7 & 28 & 28 \\
\hline
\end{tabular}

TABLE IV

Metrics for Different SCh Configurations for DT12 and US26 Networks with $|S|=19$ (MCF) and TP2

\begin{tabular}{|c|c|c|c|c|c|c|c|c|}
\hline \multirow{3}{*}{ Metric } & \multicolumn{2}{|c|}{ JoS-Gr } & \multicolumn{7}{|c|}{ SS-FON } \\
\cline { 2 - 9 } & \multicolumn{2}{|c|}{$\alpha=1$} & \multicolumn{2}{c|}{$\alpha=0$} & \multicolumn{2}{c|}{$\alpha=1$} & $\alpha_{\text {opt }}=0.04$ & $\alpha_{i}$ \\
\cline { 2 - 9 } & DT12 & US26 & DT12 & US26 & DT12 & US26 & US26 & US26 \\
\hline$\overline{n_{O C}}$ & 1 & 1.1 & 5.8 & 9.6 & 1 & 1.1 & 8.9 & 6.5 \\
\hline$\overline{n_{T R x}}$ & 11.3 & 13.7 & 5.8 & 9.6 & 11.3 & 13.7 & 9.4 & 8.8 \\
\hline$R_{S}$ & 14.5 & 19.9 & 28.4 & 29 & 14.5 & 19.9 & 29 & 29 \\
\hline
\end{tabular}

\section{Conclusions and Future Work}

In this work, we quantify the throughput scalability of Flex-Grid/SDM networks with both fixed and flexible spatial dimension given by the increment of the spatial multiplicity from 7 to 30 spatial channels. The results have been obtained for MF and equivalent MCF solutions. For spatially fixed Flex-Grid/SDM networks, we observe that network throughput scales up to a factor lower than the corresponding spatial multiplicity (4.3x versus $3.1 \mathrm{x}$-in the worst case-), because of the penalty introduced by the JoS technique. However, this solution can reduce the network cost by using less expensive and complex hardware designs at ROADMs and BVTs.

Regarding SS-FONs, we have several possibilities to configure SCh, namely, Spe-SCh, Spa-SCh and $\mathrm{S} 2-\mathrm{SCh}$. We provide a novel methodology to select the configuration based on a weighting parameter $(\alpha)$ between spectral and spatial resources. If the priority is to minimize the spectral resources per SCh, Spa-SChs or S2-SChs are configured. On the contrary, if the priority is to minimize spatial resources, Spe-SChs have to be configured. There exists an $\alpha$ value $\left(\alpha_{\text {opt }}\right)$ that 
yields the highest network throughput and reduces the cost of BVTs against pure Spe-SCh allocation. For example, if a mix of SChs (i.e., Spe-SChs, Spa-SChs and S2-SChs) is employed, the same throughput as with pure Spe-SCh allocation can be obtained, with $30-32 \%$ less laser sources and 6-9\% less transceivers per BVT.

That is, the network throughput should be contrasted with the BVTs cost, which are the major network equipment cost in SDM networks. Moreover, the spatial flexibility is achieved by a more complex ROADM design to offer space and wavelength granularity switching. Considering $\alpha_{\text {opt }}$ for SS-FONs, for 4.3x spatial multiplicity factor in either MF or MCF solutions, a 4.1x (in the worst case) scaling factor in the network throughput is obtained. The results also show that, regardless of spatial flexibility, the MCF solution is feasible given the extremely low ICXT of the current laboratory prototypes and the very small differences in terms of network throughput for all cases, except for the 19-core MCF [8]. This prototype is expected to be improved with a fabrication technology and design like the 22-core MCF, which provides better network performance. Differences from 0 to $52 \%$ in network throughput between spatially fixed Flex-Grid/SDM networks and SS-FONs with equivalent design (and cost) in BVTs are reported. In any case, a trade-off between network cost and throughput (as cost-benefit function) has to be analyzed in detail to propose an overall optimal solution in a planning scenario, which we pretend to address in a future work. Another interest aspect to be considered in a future study will be to assess dynamic SCh configurations; for example, exploring a wide range of $\alpha$ values for a same traffic demand. Finally, taking into account that survivability is a major concern in optical networks, different schemes and designs could be also studied for Flex-Grid/SDM optical core networks.

\section{ACKNOWLEDGEMENTS}

This work has been partially funded by the Spanish National Project SUNSET (TEC2014-59583C2-1-R), which receives funding from FEDER.

Rubén Rumipamba is recipient of a full scholarship from Secretaría Nacional de Ciencia y Tecnología (SENESCYT) - Ecuador (2015-AR2Q9065).

\section{REFERENCES}

[1] O. Gerstel, M. Jinno, A. Lord, and S. J. Ben Yoo, "Elastic optical networking: A new dawn for the optical layer?," IEEE Commun. Mag., vol. 50, no. 2, pp. 12-20, 2012.

[2] P. J. Winzer, "Making spatial multiplexing a reality," Nat. Photonics, vol. 8, no. 5, pp. 345-348, Apr. 2014.

[3] International Telecommunication Union - ITU-T, "G.694.1 (02/2012), Spectral grids for WDM applications: DWDM frequency grid," Ser. G.694.1, pp. 1-16, 2012.

[4] P. J. Winzer, "Spatial multiplexing: The next frontier in network capacity scaling," 39th European Conference and Exhibition on Optical Communication (ECOC 2013), London, 2013, pp. 1-4.

[5] D. J. Richardson, J. M. Fini, and L. E. Nelson, "Space Division Multiplexing in Optical Fibres," Nat. Photonics, vol. 7, pp. 354-362, 2013.

[6] J. Sakaguchi, Y. Awaji, N. Wada, A. Kanno, T. Kawanishi, T. Hayashi, T. Taru, T. Kobayashi, and M. Watanabe, "Space division multiplexed transmission of 109-Tb/s data signals using homogeneous seven-core fiber," J. Light. Technol., vol. 30, no. 4, pp. 
658-665, 2012.

[7] A. Sano, H. Takara, T. Kobayashi, H. Kawakami, H. Kishikawa, T. Nakagawa, Y. Miyamoto, Y. Abe, H. Ono, K. Shikama, M. Nagatani, T. Mori, Y. Sasaki, I. Ishida, K. Takenaga, S. Matsuo, K. Saitoh, M. Koshiba, M. Yamada, H. Masuda, and T. Morioka, "409-Tb/s + 409-Tb/s crosstalk suppressed bidirectional MCF transmission over $450 \mathrm{~km}$ using propagation-direction interleaving.," Opt. Express, vol. 21, no. 14, pp. 1677716783, 2013.

[8] J. Sakaguchi, W. Klaus, B. J. Puttnam, J. M. D. Mendinueta, Y. Awaji, N. Wada, Y. Tsuchida, K. Maeda, M. Tadakuma, K. Imamura, R. Sugizaki, T. Kobayashi, Y. Tottori, M. Watanabe, and R. V Jensen, "19-core MCF transmission system using EDFA with shared core pumping coupled via free-space optics.," Opt. Express, vol. 22, no. 1, pp. 90-5, 2014.

[9] B. J. Puttnam, R. S. Luis, W. Klaus, J. Sakaguchi, J.-M. Delgado Mendinueta, Y. Awaji, N. Wada, Y. Tamura, T. Hayashi, M. Hirano, and J. Marciante, "2.15 Pb/s transmission using a 22 core homogeneous single-mode multi-core fiber and wideband optical comb," 2015 European Conference on Optical Communication (ECOC), Valencia, 2015, pp. 1-3.

[10] Y. Amma, Y. Sasaki, K. Takenaga, S. Matsuo, J. Tu, K. Saitoh, M. Koshiba, T. Morioka, and Y. Miyamoto, "High-density multicore fiber with heterogeneous core arrangement," 2015 Optical Fiber Communications Conference and Exhibition (OFC), Los Angeles, CA, 2015, pp. 1-3.

[11] R. Rumipamba-Zambrano, J. Perelló, J. M. J. M. Gené, and S. Spadaro, "Cost-effective spatial super-channel allocation in Flex-Grid/MCF optical core networks," Opt. Switch. Netw., vol. 27, pp. 93-101, Jan. 2018.

[12] D. M. Marom and M. Blau, "Switching solutions for WDM-SDM optical networks," IEEE Commun. Mag., vol. 53, no. 2, pp. 60-68, 2015.

[13] N. Amaya, M. Irfan, G. Zervas, R. Nejabati, D. Simeonidou, J. Sakaguchi, W. Klaus, B. J. Puttnam, T. Miyazawa, Y. Awaji, N. Wada, and I. Henning, "Fully-elastic multi-granular network with space/frequency/time switching using multi-core fibres and programmable optical nodes," Opt. Express, vol. 21, no. 7, p. 8865, Apr. 2013.

[14] F.-J. Moreno-Muro, R. Rumipamba-Zambrano, P. Pavon-Marino, J. Perello, J. M. Gene, and S. Spadaro, "Evaluation of core-continuity-constrained ROADMs for flex-grid/MCF optical networks," J. Opt. Commun. Netw., vol. 9, no. 11, 2017.

[15] L. E. Nelson, M. D. Feuer, K. Abedin, X. Zhou, T. F. Taunay, J. M. Fini, B. Zhu, R. Isaac, R. Harel, G. Cohen, and D. M. Marom, "Spatial superchannel routing in a two-span ROADM system for space division multiplexing," J. Light. Technol., vol. 32, no. 4, pp. 783-789, 2014.

[16] D. M. Marom, P. D. Colbourne, A. D'Errico, N. K. Fontaine, Y. Ikuma, R. Proietti, L. Zong, J. M. Rivas-Moscoso, and I. Tomkos, "Survey of Photonic Switching Architectures and Technologies in Support of Spatially and Spectrally Flexible Optical Networking [Invited]," J. Opt. Commun. Netw., vol. 9, no. 1, p. 1, Jan. 2017.

[17] M. Klinkowski, P. Lechowicz, and K. Walkowiak, "Survey of resource allocation schemes and algorithms in spectrally-spatially flexible optical networking," Opt. Switch. Netw., vol. 27, pp. 58-78, Jan. 2018.

[18] I. Tomkos, B. Shariati, J. M. Rivas-Moscoso, D. M. Marom and D. Klonidis, "New frontiers in optical communication networking," 2017 19th International Conference on 
Transparent Optical Networks (ICTON), Girona, 2017, pp. 1-2.

[19] D. Klonidis, F. Cugini, O. Gerstel, M. Jinno, V. Lopez, E. Palkopoulou, M. Sekiya, D. Siracusa, G. Thouénon, and C. Betoule, "Spectrally and spatially flexible optical network planning and operations," IEEE Commun. Mag., vol. 53, no. 2, pp. 69-78, 2015.

[20] P. Sayyad Khodashenas, J.-M. Rivas-Moscoso, D. Siracusa, F. Pederzolli, B. Shariati, D. Klonidis, E. Salvadori, and I. Tomkos, "Comparison of Spectral and Spatial SuperChannel Allocation Schemes for SDM Networks," in Journal of Lightwave Technology, vol. 34, no. 11, pp. 2710-2716, June 2016.

[21] P. J. Winzer, "High-Spectral-Efficiency Optical Modulation Formats," J. Light. Technol., vol. 30, no. 24, pp. 3824-3835, Dec. 2012.

[22] P. Lechowicz, K. Walkowiak and M. Klinkowski, "Selection of spectral-spatial channels in SDM flexgrid optical networks," 2017 International Conference on Optical Network Design and Modeling (ONDM), Budapest, 2017, pp. 1-6.

[23] F. Pederzolli, D. Siracusa, B. Shariati, J. M. Rivas-Moscoso, E. Salvadori, and I. Tomkos, "Improving Performance of Spatially Joint-Switched Space Division Multiplexing Optical Networks via Spatial Group Sharing," J. Opt. Commun. Netw., vol. 9, no. 3, p. B1, Mar. 2017.

[24] R. Rumipamba-Zambrano, J. Perelló, J. M. Gené and S. Spadaro, "Capacity quantification of joint-switching-enabled flex-grid/SDM optical backbone networks," 2017 Optical Fiber Communications Conference and Exhibition (OFC), Los Angeles, CA, 2017, pp. 1-3.

[25] J. Y. Yen, "Finding the K shortest loopless paths in a network," Manage. Sci., vol. 17, no. 11, Jul. 1971.

[26] J. M. Rivas-Moscoso, B. Shariati, A. Mastropaolo, D. Klonidis and I. Tomkos, "Cost Benefit Quantification of SDM Network Implementations based on Spatially Integrated Network Elements," ECOC 2016; 42nd European Conference on Optical Communication, Dusseldorf, Germany, 2016, pp. 1-3..

[27] R. Ryf, S. Chandrasekhar, S. Randel, D. T. Neilson, N. K. Fontaine, and M. Feuer, "Physical layer transmission and switching solutions in support of spectrally and spatially flexible optical networks," IEEE Commun. Mag., vol. 53, no. 2, pp. 52-59, Feb. 2015.

[28] P. Poggiolini, G. Bosco, A. Carena, V. Curri, Y. Jiang, and F. Forghieri, "The GN-Model of Fiber Non-Linear Propagation and its Applications," J. Light. Technol., vol. 32, no. 4, pp. 694-721, Feb. 2014.

[29] M. D. Feuer, L. E. Nelson, X. Zhou, S. L. Woodward, R. Isaac, Benyuan Zhu, T. F. Taunay, M. Fishteyn, J. M. Fini, and M. F. Yan, "Joint Digital Signal Processing Receivers for Spatial Superchannels," IEEE Photonics Technol. Lett., vol. 24, no. 21, pp. 1957-1960, Nov. 2012. 